\title{
Equivariant slices for symplectic cones
}

\author{
Travis Schedler*
}

November 13, 2018

\begin{abstract}
The Darboux-Weinstein decomposition is a central result in the theory of complex Poisson (degenerate symplectic) varieties, which gives a local decomposition at a point as a product of the formal neighborhood of the symplectic leaf through the point and a formal slice.

Recently, conical symplectic resolutions, and more generally, Poisson cones, have been very actively studied in representation theory and algebraic geometry. This motivates asking for a $\mathbf{C}^{\times}$-equivariant version of the Darboux-Weinstein decomposition.

In this paper, we develop such a theory, prove basic results on their existence and uniqueness, study examples (quotient singularities and hypertoric varieties), and applications to noncommutative algebra (their quantization). We also pose some natural questions on existence and quantization of $\mathbf{C}^{\times}$-actions on slices to conical symplectic leaves.
\end{abstract}

\section{Introduction}

A conical complex symplectic or Poisson variety is one which is equipped with a contracting $\mathbf{C}^{\times}$-action which does not necessarily preserve the symplectic form or Poisson bracket, but rather rescales it.

Recently, the theory of conical complex symplectic resolutions $\left(\mathbf{C}^{\times}\right.$-equivariant resolutions of singularities of a cone by a symplectic variety), and more generally, Poisson cones, has been widely studied not only in mathematics, but also in physics, and has applications and connections to representation theory, symplectic geometry, quantum cohomology, mirror symmetry, equivariant cohomology, and other subjects (see, e.g., [BPW12, BLPW] for an overview of some of these). We remark that these are very special varieties: for example, conical symplectic resolutions were shown recently to be rigid (in fact, that there are finitely many of a given dimension and bound on degree), in [Nam14.

One of the fundamental tools in the study of complex Poisson (or degenerate symplectic) varieties (not necessarily conical) is the Darboux-Weinstein theorem ([Wei83]; see also

${ }^{*}$ Department of Mathematics, University of Texas at Austin; 1 University Station C1200, Austin, TX 78712-0257; trasched@gmail.com 
[Kal06, Proposition 3.3]), which gives a local structure for such varieties. Namely, recall that a symplectic leaf $Z$ of such a variety $X$ is defined as a maximal connected locally closed subvariety on which the tangent space $T_{z} Z$ is equal to the span of the Hamiltonian vector fields at $z$, for all $z \in Z$. Then the Darboux-Weinstein theorem says that a formal neighborhood $\hat{X}_{z}$ of $z \in Z$ splits as a product $\hat{Z}_{z} \times S$, for some formal transverse slice $S$ to $Z$ at $z$. This has been of fundamental use throughout the literature, perhaps even more so recently, in understanding the structure of the varieties and their quantization. For example, one can attach to the irreducible representations of the quantization their support, which are the closures of symplectic leaves. This allows one to apply geometry of $X$ to the representation theory of its quantization. This idea perhaps appeared first in Lie theory, where one can attach to each irreducible representation of a semisimple Lie algebra a nilpotent (co)adjoint orbit, which is the associated graded ideal of its kernel (i.e., the associated primitive ideal) in the universal enveloping algebra, and study the primitive ideals with fixed support. Losev showed in, e.g., [Los10, Los12], that, by quantizing the Darboux-Weinstein decomposition, one can relate irreducible representations with a given support to finite-dimensional representations of the quantization of the slice $S$.

The main idea of the present paper is to replace a formal neighborhood of a point $z \in X$ by a formal neighborhood of the punctured line $\mathbf{C}^{\times} \cdot z$, which allows one to generalize the Darboux-Weinstein decomposition to a $\mathbf{C}^{\times}$-equivariant one.

We say that $X$ admits a symplectic resolution if $X$ is normal and there is a projective resolution of singularities $\tilde{X} \rightarrow X$ with $\tilde{X}$ symplectic. A significantly weaker condition is that $X$ be a symplectic singularity [Bea00, Definition 1.1]: this requires that $X$ be normal, symplectic on its smooth locus, and that the pullback of the symplectic form under any (equivalently, every) resolution $\tilde{X} \rightarrow X$ extend to a regular (but possibly degenerate) twoform on $\tilde{X}$.

Let $\Delta^{m}:=\operatorname{Spf} \mathbf{C} \llbracket x_{1}, \ldots, x_{m} \rrbracket$ be the formal disk (the reader preferring the analytic setting could instead work with a small $m$-disk, modifying the statements accordingly).

Theorem 1. Let $X$ be a complex variety with a $\mathbf{C}^{\times}$-action and a homogeneous Poisson structure of degree $-k$. Let $Y$ be a $\mathbf{C}^{\times}$-stable leaf 1$]$ and $y \in Y$ a point with trivial stabilizer under $\mathbf{C}^{\times}$. Then:

(i) There is a decomposition of $\mathbf{C}^{\times}$-formal schemes,

$$
\hat{X}_{\mathbf{C}^{\times} \cdot y} \cong \hat{Y}_{\mathbf{C}^{\times} \cdot y} \times S,
$$

for some formal scheme $S$ (equipped here with a trivial $\mathbf{C}^{\times}$action).

(ii) $S$ is equipped with a canonical Poisson structure, and $\hat{Y}_{\mathbf{C}^{\times} \cdot y} \cong \mathbf{C}^{\times} \times \Delta^{\operatorname{dim} Y-1}$, equipped with a standard symplectic structure (see Theorem 19 below).

(iii) If $X$ admits a symplectic resolution (or more generally is a symplectic singularity in the sense of [Bea00]), then the isomorphism class of $\hat{X}_{\mathbf{C}^{\times} \cdot y}$ as a formal $\mathbf{C}^{\times}$-Poisson scheme is uniquely determined by the Poisson isomorphism class of $S$ and the dimension of $X$.

\footnotetext{
${ }^{1}$ If $X$ is a union of finitely many symplectic leaves (e.g., under assumption (iii)), then this hypothesis is unnecessary, since homogenity of the Poisson structure implies that all symplectic leaves are all $\mathbf{C}^{\times}$-stable.
} 
(iv) If the condition in (iii) holds and also $S$ admits a $\mathbf{C}^{\times}$action giving its Poisson structure degree $-k$, then there is a $\mathbf{C}^{\times}$-Poisson isomorphism of the form (2), $\hat{X}_{\mathbf{C}^{\times} \cdot y} \cong \hat{Y}_{\mathbf{C}^{\times} \cdot y} \times$ $S$ (i.e., the Poisson bivector on $\hat{X}_{\mathbf{C}^{\times} \cdot y}$ is actually the sum of the canonical Poisson bivectors on $\hat{Y}_{\mathbf{C}^{\times} \cdot y}$ and on $\left.S\right) 2$ In this case, $S$ is Poisson isomorphic to every slice appearing in an ordinary Darboux-Weinstein decomposition, $\hat{X}_{y} \cong \hat{Y}_{y} \times S$.

The theorem is proved in $\$ 3.3$, using results from 392 , We also give a quantization of the above result (Theorem 48), where we show that every $\mathbf{C}^{\times}$-compatible quantization of $X$ admits a direct-product decomposition parallel to the above.

We actually prove stronger (although more technical) versions of the above theorem. In particular, the assumption of (iii) can be relaxed to requiring that every Poisson vector field on $S$ ia Hamiltonian (the infinitesimal analogue of the condition that every symplectic torus action is Hamiltonian), as explained in Corollary 41, In Theorem 43, we explain why this latter condition is implied when $X$ is a symplectic singularity, and hence when it admits a symplectic resolution. The assumption that the stabilizer of $y$ be trivial is not strictly necessary, but is an important simplification; see Corollary 37 for a couple statements without this assumption.

As we explain in \$3, the hypotheses of (iii) and (iv) arise naturally. In particular, Examples 31 and 32 are simple typical cases where assumption (iii) does not hold and the conclusions of (iii) and (iv) fail. Indeed, in the more technical Theorem 34,(iii) below, we will describe the Poisson structure on $\hat{X}_{\mathbf{C}^{\times} \cdot y}$ guaranteed in part (iii) above, which is a sum not merely of terms coming from both $\hat{Y}_{\mathbf{C}^{\times} \cdot y}$ and $S$, but a third term, which in general represents an obstruction to writing $\hat{X}_{\mathbf{C}^{\times} \cdot y}$ as a product as in part (iv) above. Nonetheless, assumptions (iii) and (iv) are not necessary for the conclusions to hold: for example, the conclusion of (iv) holds in the case $X=\mathfrak{s l}(2)^{*}$, as explained in the next subsection, even though this case does not satisfy the assumptions of (iii) (hence neither of (iv)).

On the other hand, we explain a conjecture that implies that the assumption (iv) is unnecessary when $X$ is conical. Namely, if $X$ is conical and admits a symplectic resolution (or more generally is a symplectic singularity), is a conjecture of Kaledin [Kal09, Conjecture 1.8] that, for every $x \in X$, the ordinary Darboux-Weinstein slice $S$ at $x$ is conical (this holds in cases of interest such as hypertoric and quiver varieties, Kostant-Slodowy slices of the nilpotent cone, and linear quotient singularities). The theorem above motivates the following natural strengthening:

Question 3. Suppose $X$ is conical and admits a $\mathbf{C}^{\times}$-equivariant symplectic resolution with homogeneous symplectic form. Then for every point $x \in X$ with trivial stabilizer under $\mathbf{C}^{\times}$:

1. Does the $S$ appearing in (2) admit a contracting $\mathbf{C}^{\times}$-action?

2. Can the action be taken to make the Poisson structure on $S$ homogeneous?

\footnotetext{
${ }^{2}$ Note that this isomorphism differs slightly from the one in (i), as required since the $\mathbf{C}^{\times}$-action on $S$ has changed; however, they become the same after including either factor or after projecting to the first factor.
} 
3. Does this generalize to the situation where $X$ need not admit a symplectic resolution, but is a symplectic singularity (whose smooth locus has a homogeneous symplectic form)?

Note that it is automatic in the conical case that the degree of the generic symplectic form is positive (i.e., the degree of the Poisson bracket is negative). A positive answer to the question would imply that, for $X$ conical, the assumption in Theorem 1. (iv) holds automatically (assuming still (iii)). We will pose a quantum version of the above in Question 51 below.

Remark 4. In fact, as suggested by Namikawa, one can generalize the question to require only that $X$ be normal and conical and have a sympletic form on the smooth locus of positive degree, and not to require that $X$ be a symplectic singularity. Moreover, as he pointed out, in this case, if the slices to all symplectic leaves are conical and have symplectic forms on their smooth locus of positive degree, then one can deduce à fortiori that $X$ is a symplectic singularity (by the proof of Lemma 2.4 of [Nam13a]).

In this paper, we show that these questions have affirmative answers for linear quotient singularities (which rarely admit symplectic resolutions, as explained in [Bel09, BS13] and references therein) and that (1) and (2) have affirmative answers for hypertoric varieties, and we explicitly compute the decompositions. For the case where $X$ is the nilpotent cone of a semisimple Lie algebra (or its Kostant-Slodowy slices), see Remark 11 for a discussion.

Remark 5. It might be tempting to ask a stronger question than (2) above: Can $S$ be taken to have a $\mathbf{C}^{\times}$-action so that its Poisson structure has the same degree as that of $X$ ? However, the answer to this is negative in general. For example (cf. Remark 11 below), suppose $\mathfrak{g}$ is a semisimple Lie algebra and equip $\mathfrak{g}^{*}$ with its standard Poisson bracket of degree -1 , defined by $\mathcal{O}\left(\mathfrak{g}^{*}\right)=$ Sym $\mathfrak{g}$. Let $X \subseteq \mathfrak{g}^{*}$ be the cone of elements $\langle x,-\rangle$ where $x \in \mathfrak{g}$ is ad-nilpotent and $\langle-,-\rangle$ is the Killing form (i.e., the cone of elements whose coadjoint orbit is stable under dilation). Then $X$ is a closed Poisson subvariety, so has a Poisson bracket of degree -1 . Let $Y \subseteq X$ be any coadjoint orbit (which is automatically conical). Then, a transverse slice to the orbit is given by the Kostant-Slodowy slice in $\mathfrak{g}$ intersected with the nilpotent cone. The latter has a well-known action, called the Kazhdan action, making it a Poisson cone with bracket, of degree -2 . The Kazhdan action admits a square root, giving the Poisson bracket degree -1 , if and only if $e$ is even (i.e., for some $\mathfrak{s l}(2)$-triple $(e, h, f)$, then ad $h$ has only even eigenvalues). Note that $X$ does admit a $\mathbf{C}^{\times}$-equivariant symplectic resolution, the well-known Springer resolution.

When $e$ is not even, in general no $\mathbf{C}^{\times}$action exists giving the Poisson bracket degree -1 : for example, in the case when $e$ is a subregular nilpotent and $\mathfrak{g}=\mathfrak{s l}(n)$, then the slice is $\mathbf{C}[x, y, z] /\left(x y+z^{n}\right)$, with Poisson bracket $\{x, y\}=n z^{n-1},\{z, x\}=x,\{y, z\}=y$, and when $n$ is odd, there is no grading giving the bracket degree -1 .

We apply these techniques in particular to the cases of quotient singularities (Section 4) and hypertoric varieties (Section 5). In these cases, we give explicit equivariant DarbouxWeinstein decompositions, which are Zariski local in the hypertoric case (and sometimes 
in the quotient case, but more generally étale local). These decompositions quantize to give tensor product decompositions of the noncommutative deformations, which unlike in previous literature now incorporate the $\mathbf{C}^{\times}$-action: this means one obtains isomorphisms of filtered algebras, or alternatively graded $\mathbf{C} \llbracket \hbar \rrbracket$-algebras.

We mention one of the motivations for Theorem 1; a weak version, using only infinitesimal $\mathbf{C}^{\times}$-actions (i.e., Euler vector fields) appeared in the recent paper [PS16]. In the infinitesimal form, the result is immediate from the usual Darboux-Weinstein theorem. In [PS16], this was employed to study the structure of a canonical $\mathcal{D}$-module on a Poisson variety ES10, ES12, ES16] which represents invariants under Hamiltonian flow. Namely, the latter $\mathcal{D}$-module admits a Jordan-Hölder decomposition whose composition factors are local systems on the leaves, and in certain cases, using Theorem 1 (or its infinitesimal version), one can show that it is a direct sum of intermediate extensions of explicit weakly equivariant local systems on these leaves ([PS16, Theorem 5.1]). In Corollary 45 and Remark 47 below, we describe the local equivariant structure of this $\mathcal{D}$-module without requiring the hypotheses of [PS16, Theorem 5.1] (which are only needed to express the global structure as the aforementioned direct sum).

We begin the paper, in 92 , with easier, but still to our knowledge new, fundamental results on the Darboux theorem for $\mathbf{C}^{\times}$-equivariant symplectic structures on smooth varieties with a nontrivial $\mathbf{C}^{\times}$-action. We show that, if we formally localize along a punctured line $\mathbf{C}^{\times} \cdot x$, the resulting formal $\mathbf{C}^{\times}$-Poisson scheme is completely classified by the degree of the symplectic form and the dimension of the vector space, and give the explicit formula for the structure. Then, using this section, we proceed to our main results in $\$ 3$ and to examples and applications in 894 .

It should also be possible to give explicit formulas for the corresponding decomposition for Slodowy slices, quiver varieties, and more generally for Hamiltonian reductions of symplectic vector spaces or varieties (as well as to answer Question 3 above in these cases). The quantizations of these would yield decompositions for $U \mathfrak{g}$ and more generally for $W$-algebras, as well as for quantized quiver varieties and quantum Hamiltonian reductions.

\subsection{The case of $\mathfrak{s l}(2)$ and semisimple Lie algebras}

Let us illustrate our decomposition result in a simple example: $X=\mathfrak{g}^{*}$ for $\mathfrak{g}=\mathfrak{s l}_{2}(\mathbf{C})$. This is equipped with a standard Poisson structure, which is given by the Lie bracket: the bracket on $\mathcal{O}(X)=$ Sym $\mathfrak{g}$ is the unique extension of the Lie bracket on $\mathfrak{g}$ satisfying the Leibniz rule, $\{f g, h\}=g\{f, h\}+f\{g, h\}$. The symplectic leaves are the coadjoint orbits under $G=\mathrm{SL}_{2}(\mathbf{C})$ (which are equipped with the symplectic forms $\omega_{G \cdot \phi}\left(\left.a(x)\right|_{\phi},\left.a(y)\right|_{\phi}\right)=\phi([x, y])$, for $\phi \in \mathfrak{g}^{*}$, $x, y \in \mathfrak{g}$, and $a(x)$ and $a(y)$ the vector fields of the infinitesimal adjoint action of $\mathfrak{g}$ on $\mathfrak{g}^{*}$ ).

Although this does not satisfy the hypotheses of Theorem 1.(iii), we can still give its decomposition (e.g., Theorem 34 below still applies).

We are interested in decompositions along $\mathbf{C}^{\times}$-stable symplectic leaves other than the vertex, and the only such leaf is the unique nontrivial nilpotent coadjoint orbit, $G \cdot \chi$, for $\chi=\langle e,-\rangle$ (with $\langle-,-\rangle$ the Killing form). To obtain a $\mathbf{C}^{\times}$-equivariant decomposition, we can formally localize along the punctured line $\mathbf{C}^{\times} \cdot \chi$. Let $c=2 e f+\frac{1}{2} h^{2}$ be the Poisson 
central element, which has degree two. Then we have

$$
\begin{aligned}
\widehat{\mathcal{O}(X)}_{\mathbf{C}^{\times} \cdot \chi}=\mathbf{C}\left[f, f^{-1}\right] \llbracket e, h \rrbracket \cong \mathbf{C}\left[f, f^{-1}\right] \llbracket h \rrbracket \hat{\otimes} \mathbf{C} \llbracket c \rrbracket \\
\\
\cong \mathcal{O}\left(\widehat{\mathbf{A}^{2}} \mathbf{C}^{\times} \cdot \chi\right. \\
\end{aligned}
$$

which is a $\mathbf{C}^{\times}$-equivariant Poisson decomposition.

Remark 7. Note the subtlety that, to get a $\mathbf{C}^{\times}$-Poisson direct product decomposition, the slice $\mathbf{C} \llbracket c \rrbracket$ had to be generated in degree two.

In fact, in this case we have a much stronger statement: the decomposition above holds Zariski locally:

$$
\mathcal{O}(X \backslash\{f=0\})=\mathbf{C}\left[f, f^{-1}, h\right] \otimes \mathbf{C}[c] \cong \mathcal{O}\left(\left(\mathbf{C}^{\times} \times \mathbf{A}^{1}\right) \times \mathbf{A}^{1}\right),
$$

which is already a $\mathbf{C}^{\times}$-Poisson isomorphism.

To obtain a filtered quantization, we first give a filtered quantization of $\mathcal{O}(X)\left[f^{-1}\right]$. This is given by the Ore localization $U \mathfrak{g}\left[f^{-1}\right]$, once we verify that $S=\left\{f^{m} \mid m \geq 1\right\}$ is a right denominator set (in the terminology of [Lam99, §10], which also explains why this is sufficient to obtain an Ore localization). First, we need to demonstrate the right Ore condition: for all $a \in U \mathfrak{g}$ and all $f^{i} \in S$, there exists $b \in U \mathfrak{g}$ and $f^{j} \in S$ satisfying $a f^{j}=f^{i} b$. It suffices to prove this when $a$ ranges over algebra generators of $U \mathfrak{g}$, so for $a \in \mathfrak{g}$; in this case, setting $j=i+1$, we have $a f^{i+1}=f^{i}(f a+[a, f])$, as desired. Next, we need to verify the right reversibility condition: if $a \in U \mathfrak{g}$ satisfies $f^{i} a=0$ for some $f^{i} \in S$, then there exists $f^{j} \in S$ with $a f^{j}=0$. This is satisfied trivially since $U \mathfrak{g}$ is a domain (it has no zerodivisors).

Now, let $C:=e f+f e+\frac{1}{2} h^{2} \in U \mathfrak{g}$ be the Casimir element. Then we obtain the decomposition:

$$
U \mathfrak{g}\left[f^{-1}\right]=\mathbf{C}\left\langle f, f^{-1}, h\right\rangle /([h, f]+2 f) \otimes \mathbf{C}[C]=\mathbf{C}\left\langle x, x^{-1}, y\right\rangle /([x, y]-x) \otimes \mathbf{C}[C],
$$

with $x=f$ and $y=\frac{1}{2} h$, with $x$ and $y$ in filtered degree one and $C$ in filtered degree two. For a graded deformation quantization, letting $U_{\hbar} \mathfrak{g}:=T \mathfrak{g} \llbracket \hbar \rrbracket /(x y-y x-\hbar[x, y])_{x, y \in \mathfrak{g}}$, we get

$$
U_{\hbar} \mathfrak{g}\left[f^{-1}\right]=\mathbf{C}\langle x, y\rangle \llbracket \hbar \rrbracket /([x, y]-\hbar x) \hat{\otimes} \mathbf{C}[C],
$$

which is graded with $|\hbar|=|x|=|y|=1$ and $|C|=2$. We can also invert $\hbar$ and get a decomposition over the Laurent field $\mathbf{C}((\hbar))$.

If we are interested in quantizations of the nilpotent cone, $\operatorname{Spec} \mathcal{O}(X) /(c)$, we can divide (9) by the ideal $(C-\lambda)$ (or (10) by $\left(C-\lambda \hbar^{2}\right)$ ) for $\lambda \in \mathbf{C}$, and we recover the fact that inverting $f$ in every quantization $U \mathfrak{g} /(C-\lambda)$ yields the algebra of differential operators on $\mathbf{C}^{\times}$.

Remark 11. We believe that the above can be generalized to arbitrary semisimple $\mathfrak{g}$ in the following way. We are interested in the Poisson variety $\mathfrak{g}^{*}$, under dilation action, which gives the Poisson bracket degree -1 . The symplectic leaves are the coadjoint orbits. We consider 
such an orbit closed under the $\mathbf{C}^{\times}$-action, say $G \cdot \chi$ for $\chi \in \mathfrak{g}^{*}$. Then there is a standard construction of a transverse slice to this orbit: For $\langle-,-\rangle$ the Killing form, let $e \in \mathfrak{g}$ be such that $\langle e, x\rangle=\chi(x)$ for all $x \in \mathfrak{g}$; then $e$ is ad-nilpotent. The Jacobson-Morozov theorem guarantees the existence of $h, f \in \mathfrak{g}$ such that $(e, h, f)$ generate a subalgebra of $\mathfrak{s l}_{2}$. To this is associated a transverse slice $S:=\chi+\operatorname{ker}\left(\operatorname{ad}^{*} f\right)$, with $\left(\operatorname{ad}^{*} x\right)(\phi):=\phi \circ \operatorname{ad}(-x)$. The tangent space to the orbit $G \cdot \chi$ can be described as $V^{*}$, for $V:=[f, \mathfrak{g}] \subseteq \mathfrak{g}$, equipped with the symplectic form $\omega_{V}(x, y)=\chi([x, y])$.

There is a canonical $\mathbf{C}^{\times}$action on $\mathfrak{g}^{*}$ which preserves $S$ and restricts there to a contracting action to $\chi$, called the Kazhdan action, given by $\lambda \cdot \phi=\lambda^{-2} \lambda^{\text {ad }^{*} h}(\phi)$ for $\lambda \in \mathbf{C}^{\times}$and $\phi \in S$. However, this gives the Poisson bivector degree -2 , unlike the standard dilation action on $\mathfrak{g}^{*}$ above. To fix this, we assume that $e$ is even, which means that $\operatorname{ad}(h)$ acts only with even eigenvalues. Then, the Kazhdan action admits a square root, $\lambda \mapsto \lambda^{-1} \lambda^{\frac{1}{2} \mathrm{ad}^{*} h}$. Using this action, the condition of Theorem 1. (iv) on the degree of the Poisson bivector is satisfied 3

Then, we have a $\mathbf{C}^{\times}$-equivariant isomorphism (a $\mathbf{C}^{\times}$-equivariant, quasiclassical analogue of [Los10, Theorem 1.2.1]):

$$
\widehat{\mathfrak{g}}_{\mathbf{C}^{\times} \cdot \chi} \cong \widehat{V^{*}} \mathbf{C}^{\times} \cdot \chi \times \hat{S}
$$

Following [Los10], this yields a decomposition of a localization of a certain completion of the enveloping algebra $U_{\hbar} \mathfrak{g}$. Let $\mathfrak{g}(i) \subseteq \mathfrak{g}$ denote the $i$-weight space of ad $h$. Equip $\mathfrak{g}(-1)$ with the symplectic form $(x, y)=\chi([x, y])$, and let $\mathfrak{l} \subseteq \mathfrak{g}(-1)$ be a Lagrangian. Set $\mathfrak{m}:=$ $\bigoplus_{i \leq-2} \mathfrak{g}(i) \oplus \mathfrak{l}$, and let $\mathfrak{n}:=\mathfrak{m} \cap \operatorname{ker}(\chi)$. Then [Los10, Theorem 1.2.1] should strengthen to the following graded filtered isomorphism:

$$
\widehat{U \mathfrak{g}_{\mathfrak{n}}}\left[f^{-1}\right] \cong \mathcal{D}\left(\mathbf{C}^{\times} \times \Delta^{\operatorname{dim} Y / 2-1}\right) \hat{\otimes} \mathcal{W}_{\chi}
$$

which is compatible with the $\mathbf{C}^{\times}$-action by $\lambda \mapsto \lambda^{\text {ad } h / 2}$ on the left-hand side, and the action on the first factor on the right-hand side by dilating in the $\mathbf{C}^{\times}$direction. Here, $\mathcal{W}_{\chi}$ is the $W$ algebra quantizing $S$, which was denoted $U(\mathfrak{g}, e)$ in [Los10], and is defined as $\left(U \mathfrak{g} / U \mathfrak{g} \cdot \mathfrak{m}^{\prime}\right)^{\mathfrak{m}}$ for $\mathfrak{m}^{\prime}=\{x-\chi(x) \mid x \in \mathfrak{m}\} \subseteq U \mathfrak{g}$. We equip it with the filtration obtained by reducing by half the degrees of the Kazhdan filtration (compatible with our grading on $S$ above). From this, [Los10, Theorem 1.2.1] follows (in the case of even $e$ ) by completing along $\mathfrak{m}^{\prime}$. This also recovers the example above (8), (9), in the case $\mathfrak{g}=\mathfrak{s l}(2)$.

When we replace $X$ with the nilpotent cone $X=\operatorname{Nil}\left(\mathfrak{g}^{*}\right)$, we obtain a decomposition $\hat{X}_{\mathbf{C}^{\times} \cdot \chi} \cong{\widehat{V^{*}}}_{\mathbf{C}^{\times} \cdot \chi} \times(\widehat{S \cap X})$. In this case, the hypotheses of Theorem 11 are satisfied; the content here is the explicit identification of the slice with the Kostant-Slodowy slice $\widehat{S \cap X}$. The quantization then is the quotient of (13) by the augmentation ideal of the center of $U \mathfrak{g}$. As a corollary, we can deduce that Question 3 has a positive answer for the nilpotent cone.

\footnotetext{
${ }^{3}$ Since $S$ is an affine space, even if $e$ is not even, we can still pick some $\mathbf{C}^{\times}$-action for which the Poisson structure on $S$ has degree -1 , but this is not natural. Moreover, as explained in Remark 5 the intersection $S \cap \operatorname{Nil}\left(\mathfrak{g}^{*}\right)$ with the nilpotent cone does not in general have a $\mathbf{C}^{\times}$action giving the bracket degree -1 .
} 


\subsection{Conventions}

We will work with varieties or formal schemes over $\mathbf{C}$ ( so $\mathbf{A}^{n}=\mathbf{C}^{n}$ ). When we take a product of formal schemes, we always mean the formal scheme obtained by completing the corresponding tensor product of rings of functions, i.e., Spf $A \times \operatorname{Spf} B=\operatorname{Spf}(A \hat{\otimes} B)$, where if $A$ is given the $I$-adic topology and $B$ the $J$-adic topology (for $I \subseteq A$ and $J \subseteq B$ ideals) then $A \hat{\otimes} B$ is the completion of $A \otimes B$ in the $(I+J)$-adic topology. When we say a $\mathbf{C}^{\times}$-variety (or formal scheme), we mean a variety (or formal scheme) equipped with a $\mathbf{C}^{\times}$-action. For an affine variety, this just means that the algebra of functions is $\mathbf{Z}$-graded. When we say a $\mathbf{C}^{\times}$Poisson variety (or formal scheme), we mean one equipped with a $\mathbf{C}^{\times}$-action and a Poisson structure homogeneous for this action. A $\mathbf{C}^{\times}$-Poisson (iso)morphism is a $\mathbf{C}^{\times}$-equivariant Poisson (iso)morphism. When $f$ is a homogeneous element of a Z-graded algebra, then $|f|$ will denote its degree.

When we say "symplectic leaf," $Y$ of a Poisson variety $X$, we will always mean a algebraic symplectic leaf, i.e., $Y$ is a locally closed algebraic subvariety such that, for every $y \in Y$, the tangent space $T_{y} Y$ is the span of the restriction of all Hamiltonian vector fields (originally defined in any neighborhood of $y$ ) to $Y$. Moreover, we always assume symplectic leaves are connected and that they are maximal (i.e., they are not proper open subsets of a larger locally closed connected subvariety with the property in the previous sentence). (Note that, since we are usually only concerned with local properties, these last two conditions of maximality and connectedness will be irrelevant for most of our results.) Here is an important example: if $X$ is a union of finitely many algebraic symplectic leaves (which holds, for instance, when $X$ admits a symplectic resolution or is a symplectic singularity) then the symplectic leaves are the connected components of the loci $X_{i} \subseteq X$ of points $x \in X$ where the restriction of Hamiltonian vector fields span a subspace of dimension $i$ in $T_{x} X$.

\subsection{Acknowledgements}

I would like to thank Ivan Losev and Hiraku Nakajima for useful discussions, and Yoshinori Namikawa for his feedback on Question 3. I am grateful to the anonymous referees for important suggestions and corrections. The impetus for this work was [PS16], and I thank Nick Proudfoot for his collaboration on that project. This work was partially supported by NSF grant DMS-1406553.

\section{$2 \quad \mathrm{C}^{\times}$-equivariant formal symplectic geometry}

Given an affine $\mathbf{C}^{\times}$-variety $X$ and $x \in X$, let $\bar{x}$ denote the image of $x$ in the categorical quotient $X / / \mathbf{C}^{\times}=\operatorname{Spec} \mathcal{O}(X)^{\mathbf{C}^{\times}}$.

Lemma 14. Let $X$ be an irreducible affine variety with a faithful $\mathbf{C}^{\times}$-action, and $x \in X$ a point with trivial stabilizer. Then there is a $\mathbf{C}^{\times}$-stable affine open subvariety $U$ containing $x$ together with an isomorphism $U \cong \mathbf{C}^{\times} \times U / / \mathbf{C}^{\times}$, such that $x \mapsto(1, \bar{x})$. 
Remark 15. By Sumihiro's theorem, we can drop the assumption that $X$ is affine if we assume that it is normal, since then every orbit is contained in a $\mathbf{C}^{\times}$-stable open affine subvariety.

Proof of Lemma 14. Let $t \in \mathcal{O}(X)$ be any homogeneous function (of weight one) which restricts on the line $\mathbf{C}^{\times} \cdot x$ to a homogeneous coordinate function (of weight one by our assumption). Let $U$ be the complement of the locus where $t=0$. Then $U$ is stable under the $\mathbf{C}^{\times}$-action, so $\mathcal{O}(U)$ is spanned by homogeneous functions. Every homogeneous function is of the form $f t^{k}$ where $f$ has weight zero, i.e., $f \in \mathcal{O}(U)^{\mathbf{C}^{\times}}$, and $k$ is an integer. Thus the inclusion of algebras $\mathcal{O}(U)^{\mathbf{C}^{\times}} \otimes \mathbf{C}\left[t, t^{-1}\right] \rightarrow \mathcal{O}(U)$ is an isomorphism, i.e., $U \cong \mathbf{C}^{\times} \times U / / \mathbf{C}^{\times}$ as $\mathbf{C}^{\times}$-varieties (giving $U / / \mathbf{C}^{\times}$the trivial $\mathbf{C}^{\times}$-action).

From now on, we will use the following notation for a locally closed affine subvariety $Y$ of a (not necessarily affine) variety $X$. Let $U \subseteq X$ be an open affine subset such that $Y$ is closed in $U$ (i.e., obtained by inverting an element whose vanishing locus on $\bar{Y}$ is $\bar{Y} \backslash Y$ ). Then the completion $\hat{\mathcal{O}}(X)_{Y}$ is defined as the completion $\hat{\mathcal{O}}(U)_{Y}$, which clearly does not depend on the choice of open affine subset $U$. We set $\hat{X}_{Y}:=\operatorname{Spf} \hat{\mathcal{O}}(X)_{Y}$. We deduce the following corollary:

Corollary 16. Let $X$ be a (not necessarily affine) irreducible variety with a faithful $\mathbf{C}^{\times}$action, and $x \in X$ a point having trivial stabilizer. Then the formal neighborhood $\hat{X}_{\mathbf{C}^{\times} \cdot x}$ of $\mathbf{C}^{\times} \cdot x$ is $\mathbf{C}^{\times}$-equivariantly isomorphic to the product $\mathbf{C}^{\times} \times Z$, where $Z=\operatorname{Spf} \mathcal{O}(Z)$ is a formal affine scheme with the trivial $\mathbf{C}^{\times}$-action.

Note here that $\mathbf{C}^{\times} \times Z$ is, by definition, Spf $\mathbf{C}\left[t, t^{-1}\right] \hat{\otimes} \mathcal{O}(Z)$ (cf. 81.2). In this case, $\mathcal{O}(Z)$ is equipped with the $\mathfrak{p}$-adic topology for $\mathfrak{p} \subseteq \hat{\mathcal{O}}(Z)$ a maximal ideal, so the completed tensor product is with respect to the $(\mathfrak{p})$-adic topology.

Proof of Corollary 16. The only thing that has to be explained is how to remove the affineness assumption. The point is that $\mathbf{C}^{\times} \cdot x$ is still affine, so the completion $\hat{X}_{\mathbf{C}^{\times} \cdot x}=$ Spf $\hat{\mathcal{O}}(X)_{\mathbf{C}^{\times} \cdot x}$ is still an affine formal scheme. Now, as $\hat{\mathcal{O}}(X)_{\mathbf{C}^{\times} \cdot x}$ is the completion of a ring with a locally finite $\mathbf{C}^{\times}$-action, it it is topologically (in the $\mathfrak{p}$-adic topology, with $\mathfrak{p}$ the ideal of $\mathbf{C}^{\times} \cdot x$ as before) spanned by homogeneous elements. The same proof as before applies to show that the inclusion of algebras $\mathbf{C}\left[t, t^{-1}\right] \hat{\otimes} \hat{\mathcal{O}}(X)_{\mathbf{C}^{\times} \cdot x}^{\mathbf{C}^{\times}} \rightarrow \hat{\mathcal{O}}(X)_{\mathbf{C}^{\times} \cdot x}$ is an isomorphism.

Now, the Darboux theorem for formal neighborhoods goes through in this context. Let $\Delta$ denote the formal polydisc, i.e., the formal neighborhood of the origin in $\mathbf{A}^{1}$. For all $m \geq 1$, let $\Delta^{m}$ denote the formal $m$-polydisc, i.e., $\mathbf{S p f} \mathbf{C} \llbracket z_{1}, \ldots, z_{m} \rrbracket$. Let us call the standard symplectic structure of degree $k$ on $\mathbf{C}^{\times} \times \Delta^{2 n-1}$, coordinatized as $\operatorname{Spf} \mathbf{C}\left[t, t^{-1}\right] \llbracket u, z_{1}, \ldots, z_{2 n-2} \rrbracket$, with $u, z_{i}$ in degree zero, the following:

$$
t^{k-1} d t \wedge d u+\sum_{i=1}^{n-1} d\left(t^{k} z_{2 i-1}\right) \wedge d z_{2 i} .
$$


Let us also record the Poisson bivector in the above situation. We make the substitution $z_{2 i-1}^{\prime}:=t^{k} z_{2 i-1}$ for all $1 \leq i \leq 2 n-1$.

$$
\pi_{2 n,-k}:=t^{-k+1} \partial_{u} \wedge \partial_{t}+\sum_{i=1}^{n-1} \partial_{z_{2 i-1}^{\prime}} \wedge \partial_{z_{2 i}} .
$$

Theorem 19. Any symplectic structure on $\mathbf{C}^{\times} \times \Delta^{2 n-1}$ of degree $k$ can be taken to the standard one by a $\mathbf{C}^{\times}$-equivariant change of coordinates.

Remark 20. As pointed out by a referee, at least in the case $k \neq 0$, there is a simple interpretation and proof of this statement using contact geometry: a symplectic structure $\omega$ on $\mathbf{C}^{\times} \times \Delta^{2 n-1}$ of degree $k \neq 0$ is of the form $\omega=d p^{*}(\theta)$ where $p: \mathbf{C}^{\times} \times \Delta^{2 n-1} \rightarrow \Delta^{2 n-1}$ is the projection and $\theta \in \Omega^{1}\left(\Delta^{2 n-1}\right) \otimes O(k)$ is a contact structure on $\Delta^{2 n-1}$ equipped with the trivial bundle $O(k)$ viewed as $\mathbf{C}^{\times}$-equivariant with the weight $k$ action of $\mathbf{C}^{\times}$. Then $\theta$ can always be put in the standard form $\theta=\left(d u+\sum_{i} z_{2 i-1} d z_{2 i}\right) \otimes t^{k}$. (In other words, we can write $\omega=d\left(t^{k} p^{*} \alpha\right)$ where $\alpha \in \Omega^{1}\left(\Delta^{2 n-1}\right)$ is a usual contact one-form on $\Delta^{2 n-1}$.) Note that, when $k=0$, then $d\left(p^{*} \theta\right)=p^{*} d \theta$ which is degenerate for any one-form $\theta \in \Omega^{1}\left(\Delta^{2 n-1}\right)$, so that this proof does not seem to work.

Proof. As before, write $\mathbf{C}^{\times} \times \Delta^{2 n-1}=\operatorname{Spf} \mathbf{C}\left[t, t^{-1}\right] \llbracket u, z_{1}, \ldots, z_{2 n-2} \rrbracket$, and choose coordinates $u, z_{1}, \ldots, z_{2 n-2}$ so that the restriction of the symplectic structure $\omega$ to $t=1, u=$ $z_{1}, \ldots, z_{2 n-2}=0$ is the standard one,

$$
\left.\omega\right|_{(1,0, \ldots, 0)}=d t \wedge d u+\sum_{i=1}^{n-1} d z_{2 i-1} \wedge d z_{2 i} .
$$

Then, since it is homogeneous, the symplectic structure must have the form

$$
\omega=t^{k-1} d t \wedge d u+\sum_{i=1}^{n-1} d\left(t^{k} z_{2 i-1}\right) \wedge d z_{2 i}+\omega^{\prime},
$$

where $\omega^{\prime}$ is a closed two-form vanishing at the ideal $\left(t-1, u, z_{1}, \ldots, z_{2 n-2}\right)$. Since $\omega^{\prime}$ is homogeneous, it must vanish at the entire locus $t \neq 0, u=z_{1}=\cdots=z_{2 n-2}=0$, and hence at the ideal $\left(u, z_{1}, \ldots, z_{2 n-2}\right)$.

Now we are in a position to apply Moser's trick, as in the proof of the usual Darboux theorem. First, note that $\omega_{c}:=\omega-c \omega^{\prime}$ is closed and nondegenerate for all $c \in \mathbf{C}$. We need to show that there exists a $\mathbf{C}^{\times}$-equivariant symplectomorphism $\Phi$ such that $\Phi^{*} \omega_{1}=\omega_{0}$. To do so, first note that all closed two-forms on $\mathbf{C}^{\times} \times \Delta^{2 n-1}$ are exact, so we can write $\omega^{\prime}=d \alpha$ for some one-form $\alpha$, also of weight $k$ with respect to the $\mathbf{C}^{\times}$action. Then, we can consider for all $0 \leq c \leq 1$ the vector field $\theta_{c}$ such that $i_{\theta_{c}}\left(\omega_{c}\right)=-\alpha$. Since $\alpha$ and $\omega_{c}$ have weight $k, \theta_{c}$ will have weight zero. It moreover vanishes on $\left(u, z_{1}, \ldots, z_{2 n-2}\right)$. As a consequence, it integrates in $\mathbf{C}^{\times} \times \Delta$ to an automorphism $\Phi_{s}$ such that $\Phi_{0}=\operatorname{Id}$ and $\left.\frac{d}{d s} \Phi_{s}\right|_{s=c}=\theta_{c}$. Then the 
calculation in Moser's trick shows that $\Phi_{c}^{*} \omega_{c}=\omega_{0}$ for all $c$, and in particular that $\Phi_{1}^{*} \omega_{1}=\omega_{0}$ as desired. Namely,

$$
\frac{d}{d s}\left(\Phi_{s}^{*} \omega_{s}\right)=\Phi_{s}^{*} L_{\theta_{s}} \omega_{s}+\Phi_{s}^{*} \omega^{\prime}=\Phi_{s}^{*}\left(d i_{\theta_{s}}+i_{\theta_{s}} d\right) \omega_{s}+\Phi_{s}^{*} \omega^{\prime}=-\Phi_{s}^{*} d \alpha+\Phi_{s}^{*} \omega^{\prime}=0
$$

so $\Phi_{c}^{*} \omega_{c}$ is constant, and $\Phi_{0}=\operatorname{Id}$ implies $\Phi_{0}^{*} \omega_{0}=\omega_{0}$.

Putting the two results together, we obtain the following.

Corollary 21. Let $X$ be a smooth symplectic variety with a faithful $\mathbf{C}^{\times}$-action and a homogeneous symplectic structure of degree $k$. Then for any $x \in X$ which has trivial stabilizer, a formal neighborhood of $\mathbf{C}^{\times} \cdot x$ is isomorphic to $\mathbf{C}^{\times} \times \Delta^{\operatorname{dim} X-1}$ with the standard symplectic structure (17).

We can also deduce the classification of symplectic structures in the case that the $\mathbf{C}^{\times}$ action is not faithful:

Corollary 22. Equip $\mathbf{C}^{\times} \times \Delta^{2 n-1}$ with a $\mathbf{C}^{\times}$action which along the line $\mathbf{C}^{\times} \times\{0\}$ has the form $\lambda \cdot(t, 0)=\left(\lambda^{\ell} t, 0\right)$. Then any weight $k$ symplectic form can be written in some choice of homogeneous coordinates $\left(t, u, z_{1}, \ldots, z_{2 n-2}\right.$ ) (with $t$ invertible of weight $\ell$ and restricting to the standard coordinate on $\left.\mathbf{C}^{\times} \times\{0\}\right)$ in the standard form,

$$
d t \wedge d u+\sum_{i=1}^{n-1} d z_{2 i-1} \wedge d z_{2 i}
$$

The same holds for the completion of a smooth symplectic variety with a $\mathbf{C}^{\times}$action along a line $\mathbf{C}^{\times} \cdot x$ where the stabilizer of $x$ is the group of $\ell$-th roots of unity.

Note that there is no need to multiply the first term by a power of $t$ since now we can take $u$ to have nonzero degree (in fact, degree $k-\ell$ ).

Proof. The proof is similar to that of Theorem 19 and Lemma 14. First, we can pick $t$ to be a weight $\ell$ element mapping modulo the ideal, call it $\tilde{J}$, of $\mathbf{C}^{\times}$to the coordinate $t$. By linear algebra, a homogenous symplectic form on a graded vector space can be written in standard form in terms of a homogeneous basis, and we can take this to include any nonzero fixed homogeneous element. Thus, at $1 \in \mathbf{C}^{\times}$, we can find homogeneous cotangent vectors $\bar{u}, \bar{z}_{1}, \ldots, \bar{z}_{2 n-2}$ so that $\omega=\bar{t} \wedge \bar{u}+\cdots+\bar{z}_{2 n-1} \wedge \bar{z}_{2 n-2}$. Lifting these to homogeneous generators of the ideal $\left(u, z_{1}, \ldots, z_{2 n-2}\right)$, we obtain homogeneous coordinates $t, u, z_{1}, \ldots, z_{2 n-2}$ so that, at $1 \in \mathbf{C}^{\times}, \omega$ restricts to $d t \wedge d u+d z_{1} \wedge d z_{2}+\cdots+d z_{2 n-3} \wedge d z_{2 n-2}$. The rest of the argument is the application of Moser's trick as in the proof of Theorem 19.

Remark 24. We are interested here in the case where $x$ is not a fixed point since we are going to study symplectic leaves of $\mathbf{C}^{\times}$-Poisson varieties (with homogeneous Poisson bracket of some degree) which are stable under the $\mathbf{C}^{\times}$-action, but on which this action is nontrivial 
(the main example being Poisson cones with finitely many symplectic leaves, where all leaves other than the vertex have this form).

In the case of fixed points, the situation is different. In the case that $x$ is a fixed point such that, in some neighborhood $U$ of $X$, the limit $t \cdot y$ exists as $t \rightarrow 0$ for all $y \in U$, which is called an elliptic fixed point (as studied recently in, e.g., [BDMN]), the BialynickiBirula decomposition theorem yields an analogue of Corollary 16, i.e., that in a formal neighborhood of $x$, we have an equivariant isomorphism $\hat{X}_{x} \cong \Delta^{2 n}=\operatorname{Spf} \llbracket x_{1}, \ldots, x_{2 n} \rrbracket$ where each $x_{i}$ is homogeneous of some nonnegative degree. Then, the formal Darboux theorem applies with the same proof, where we only require equivariant changes of coordinates, so that there is an equivariant change of coordinates under which the symplectic structure is

$$
\sum_{i=1}^{n} d x_{2 i-1} \wedge d x_{2 i}
$$

of degree $\left|x_{2 i-1}\right|+\left|x_{2 i}\right|$ (which therefore cannot depend on $i$ ).

\subsection{Proof without Moser's trick}

It is instructive to give a proof of Theorem 19 using only Poisson brackets and not symplectic forms, and hence without using Moser's trick, since these will be unavailable in the singular Poisson case we consider in the sequel. Thus, the reader may wish to skip this subsection for now, and return to it before reading the proof of Theorem 34 given in $\$ 3.4$ below, which uses the same ideas. In two places, we give here a simpler, more explicit argument than the one we need in \$3.4, thereby avoiding the use of [Kal06, Lemma 3.2], although we also outline below how the latter argument would go.

Define the ideals $J:=\left(z_{1}, \ldots, z_{2 n-2}\right)$ and $\tilde{J}:=(J, u)$. Define the notation $z_{2 i-1}^{\prime}=t^{k} z_{2 i-1}$ and $z_{2 i}^{\prime}=z_{2 i}$. Then, we need to perform a coordinate change which is the identity modulo $\tilde{J}$ so that

$$
\{t, u\}=t^{1-k}, \quad\left\{z_{2 i-1}^{\prime}, z_{2 i}^{\prime}\right\}=1,
$$

and all other brackets of the $t, u, z_{1}^{\prime}, \ldots, z_{2 n}^{\prime}$ zero.

The first step is to change the $z_{i}^{\prime}$ so that $\left\{t, z_{i}^{\prime}\right\}=0$ for all $i$. As a result of this, the degree-zero part of the centralizer of $t$ will be $\mathbf{C} \llbracket z_{1}, \ldots, z_{2 n-2} \rrbracket$. We will do this explicitly in the next paragraph. We would like to point out, though, that this computation can be replaced with the following more conceptual argument: for the existence of such a coordinate change, we only need to know that the degree-zero centralizer of $t$ had this form for some different choice of $z_{i}$. To prove the latter we can alternatively follow [Kal06, Lemma 3.2] as in the proof of Theorem 34, (ii) in $\$ 3.4$ below; the idea being that $\nabla_{u}:=\{t, u\}^{-1}\{t,-\}$ defines a flat connection on $\mathcal{O}\left(\mathbf{C}^{\times} \times \Delta^{2 n-1}\right)^{\mathbf{C}^{\times}}$as an $\mathbf{C} \llbracket u \rrbracket$-module, together with its filtration by powers of the ideal of $\left(\mathbf{C}^{\times} \times\{0\}\right) / \mathbf{C}^{\times}$. Hence $\mathcal{O}\left(\mathbf{C}^{\times} \times \Delta^{2 n-1}\right) \cong \mathbf{C} \llbracket u \rrbracket \hat{\otimes}\left(\mathcal{O}\left(\mathbf{C}^{\times} \times \Delta^{2 n-1}\right)^{\mathbf{C}^{\times}}\right)^{\nabla}$, and the second factor consists of the degree-zero elements commuting with $t$. It then follows that the latter factor is a power-series algebra generated by elements which map to the same basis for its cotangent space as the original elements $z_{1}, \ldots, z_{2 n-2}$. We omit further details, since we give an explicit construction of the coordinate change needed in the next paragraph. 
To explicitly find the needed modification of the $z_{i}^{\prime}$, we proceed inductively. We know that $\left\{t, z_{i}^{\prime}\right\} \in J$ for all $i$. Suppose that $\left\{t, z_{i}^{\prime}\right\} \in J^{m}$ for some $m \geq 1$. Then we can make the coordinate change $z_{i}^{\prime} \mapsto z_{i}^{\prime \prime}:=z_{i}^{\prime}-t^{k-1} \int\left\{t, z_{i}^{\prime}\right\} d u$, where $\int f d u$ denotes the unique antiderivative of $f$ by $u$ which is a multiple of $u$. Since $\left\{t, z_{j}^{\prime}\right\} \in J$ for all $j,\{t, u\}-t^{1-k} \in J$, and $\left\{t, z_{i}^{\prime}\right\} \in J^{m}$, it follows from the definition that $\left\{t, z_{i}^{\prime \prime}\right\} \in J^{m+1}$, as desired. Moreover, $z_{i}^{\prime \prime}-z_{i}^{\prime} \in J^{m+1}$, so we can iterate the aforementioned coordinate change $z_{i}^{\prime} \mapsto z_{i}^{\prime \prime}$ and the result will converge to a function $\overline{z_{i}^{\prime}}$ such that $\left\{t, \overline{z_{i}^{\prime}}\right\}=0$. Doing this for all $i$, we obtain a change of coordinates $z_{i}^{\prime} \mapsto \overline{z_{i}^{\prime}}$ which is the identity modulo $J$, so that $\left\{t, \overline{z_{i}^{\prime}}\right\}=0$ and the original assumptions remain satisfied. From now on we assume that this is done, so that $\left\{t, z_{i}^{\prime}\right\}=0$ for all $i$.

Now, the entire subalgebra $\mathbf{C}\left[t, t^{-1}\right] \llbracket z_{1}, \ldots, z_{2 n-2} \rrbracket$ of elements commuting with $t$ must be closed under the Poisson bracket. Since the bracket has degree $-k$, it follows that the degree-zero subalgebra $\mathbf{C} \llbracket z_{1}, \ldots, z_{2 n-2} \rrbracket$ is closed under the operation $\{-,-\}^{\prime}:=t^{k}\{-,-\}$. Moreover, this operation defines on it a Poisson bracket which is symplectic. By the ordinary formal Darboux theorem, we can make a change of variables of $\mathbf{C} \llbracket z_{1}, \ldots, z_{2 n-2} \rrbracket$ so that $\{-,-\}^{\prime}$ is in standard form. As a result, $\left\{z_{i}^{\prime}, z_{j}^{\prime}\right\}$ are all what we desire.

Therefore, all of the brackets except for those with $u$ are as desired. To finish, we will show that there is a change of variables $u \mapsto u^{\prime}$ so that $\left\{u^{\prime}, z_{i}^{\prime}\right\}=0$ for all $i$, so that $u^{\prime} \equiv u$ $(\bmod J)$ and $u^{\prime}$ has weight zero. We could also apply [Kal06, Lemma 3.2] for this, to show that $\mathcal{O}\left(\mathbf{C}^{\times} \times \Delta^{2 n-1}\right)^{\mathbf{C}^{\times}}$decomposes as a tensor product of $\mathbf{C} \llbracket z_{1}, \ldots, z_{2 n-2} \rrbracket$ and its centralizer, and pick $u^{\prime}$ as a generator of the latter. This is what we must do in $\$ 3.4$ below. However, we give in the next paragraph a shortcut argument using that every Poisson vector field on a symplectic disk is Hamiltonian.

Let $\xi_{u}$ be the Hamiltonian vector field $\{u,-\}$. Since $\{u, t\}=t^{1-k}$, the Jacobi identity implies that $t^{k} \xi_{u}$ preserves $\mathbf{C} \llbracket z_{1}, \ldots, z_{2 n-2} \rrbracket$. Moreover, the Jacobi identity again proves that it is a Poisson vector field on Spf $\mathbf{C} \llbracket z_{1}, \ldots, z_{2 n-2} \rrbracket$ where the latter is equipped with the Poisson structure $\{-,-\}^{\prime}$ discussed above. Since every Poisson vector field on a symplectic disk is Hamiltonian, $t^{k} \xi_{u}=t^{k}\{f,-\}$ for some $f \in \mathbf{C} \llbracket z_{1}, \ldots, z_{2 n-2} \rrbracket$ which we can assume to be in the augmentation ideal. Then we can make the substitution $u \mapsto u-f$ which will have the desired property.

\subsection{Quantization}

The above theorem has the following consequence. Suppose that $X=\operatorname{Spec}(\mathcal{O}(X))$ is an affine Poisson variety (such as an affine symplectic variety), or that $X=\operatorname{Spf} \mathcal{O}(X)$ is the formal neighborhood of some subvariety of an affine Poisson variety.

When $X=\operatorname{Spec}\left(\mathcal{O}(X)\right.$ ), recall that a quantization $A_{\hbar}$ of $X$ (or equivalently of $\mathcal{O}(X)$ ) is a $\mathbf{C} \llbracket \hbar \rrbracket$-algebra equipped with an algebra epimorphism pr : $A_{\hbar} \rightarrow \mathcal{O}(X)$ with kernel $\hbar A_{\hbar}$, such that $A_{\hbar}$ is isomorphic, as a $\mathbf{C} \llbracket \hbar \rrbracket$-module, to $\mathcal{O}(X) \llbracket \hbar \rrbracket=\left\{\sum_{m>0} a_{m} \hbar^{m} \mid a_{m} \in \mathcal{O}(X)\right\}$, and satisfies the following additional axiom. For any $f \in \mathcal{O}(X)$, let $\tilde{f} \in A_{\hbar}$ be an arbitrary lift. Then $A_{\hbar}$ is a quantization if it additionally satisfies:

$$
\tilde{a} \star \tilde{b}-\tilde{b} \star \tilde{a} \equiv \hbar \widetilde{\{a, b\}} \quad(\bmod \hbar)^{2}, \forall a, b \in \mathcal{O}(X) ;
$$


it is easy to see that the axiom holds for one choice of lift if and only if it holds for all choices. We can make the same definition when $X=\operatorname{Spf} \mathcal{O}(X)$ is the formal spectrum of a complete topological Poisson algebra, where now $A_{\hbar}$ is also a complete topological algebra.

Next, suppose that $X$ has a $\mathbf{C}^{\times}$-action such that the Poisson structure has degree $-k$. Then we can ask that the quantization $A_{\hbar}$ be compatible with the action. This means that $A_{\hbar}$ admits an infinitesimal action of $\mathbf{C}^{\times}$, i.e., an Euler derivation $\mathrm{Eu}: A_{\hbar} \rightarrow A_{\hbar}$, which satisfies $\operatorname{Eu}(\hbar)=k \hbar$, and such that the induced action on $A_{\hbar} / \hbar A_{\hbar} \cong \mathcal{O}(X)$ agrees with the original Euler derivation $(\mathrm{Eu}(x)=|x| \cdot x$ when $x$ is homogeneous). This implies that $A_{\hbar}$ is the $\hbar$-adic completion of a graded algebra having $|\hbar|=k$. This graded algebra can be recovered as the $\mathbf{C}^{\times}$-finite part, $A_{\hbar}^{f} \subseteq A_{\hbar}$, defined as the collection of elements $a \in A_{\hbar}$ such that $\left\{\mathrm{Eu}^{k}(a)\right\}$ spans a finite vector space. Slightly abusively, we will refer to such $A_{\hbar}$ themselves as graded algebras and call an isomorphism $A_{\hbar} \rightarrow B_{\hbar}$ graded if it is compatible with the infinitesimal $\mathbf{C}^{\times}$-action, i.e., the Euler derivations.

Let $\operatorname{Weyl}_{\hbar, k}\left(\mathbf{C}^{2 n-2}\right)$ be the graded $\mathbf{C}[\hbar]$-algebra which is generated by elements $z_{1}^{\prime}, \ldots, z_{2 n-2}^{\prime}$, with relations $\left[z_{2 i-1}^{\prime}, z_{2 i}^{\prime}\right]=\hbar$ for $1 \leq i \leq n-1$ and all other $\left[z_{i}^{\prime}, z_{j}^{\prime}\right]$ zero, and equipped with the grading with $|\hbar|=k,\left|z_{2 i-1}^{\prime}\right|=k$ and $\left|z_{2 i}\right|=0$ for all $1 \leq i \leq n-1$. (Note that, if we set $z_{2 i-1}:=t^{-k} z_{2 i-1}^{\prime}$ and $z_{2 i}:=z_{2 i}^{\prime}$ for $1 \leq i \leq n-1$, we recover generators of degree zero, and this is consistent with the notation of $\$ 2.1$.)

Theorem 26. The unique $\mathbf{C}^{\times}$-compatible quantizations of $\mathbf{C}^{\times} \times \mathbf{A}^{2 n-1}$ and of $\mathbf{C}^{\times} \times \Delta^{2 n-1}$, equipped with the standard symplectic structures (17), are given by the completions of the graded algebra (for $|t|=1$ and $|u|=0$ ):

$$
\mathcal{D}_{\hbar, k}\left(\mathbf{C}^{\times} \times \mathbf{A}^{n-1}\right):=\mathbf{C}\langle t, u\rangle[\hbar]\left[t^{-1}\right] /\left([t, u]-\hbar t^{1-k}\right) \otimes_{\mathbf{C}[\hbar]} \operatorname{Weyl}_{\hbar, k}\left(\mathbf{C}^{2 n-2}\right)
$$

with respect to the ideal $(\hbar)$ and the ideal generated by $\hbar, u, z_{1}^{\prime}, \ldots, z_{2 n-2}^{\prime}$, respectively.

Note that, for $k=1, \mathcal{D}_{\hbar, k}\left(\mathbf{C}^{\times} \times \mathbf{A}^{n-1}\right)$ is the Rees algebra of the ring of differential operators on $\mathbf{C}^{\times} \times \mathbf{A}^{n-1}$; for general $k$, the ring of differential operators is recovered by setting $\hbar=1$. Also, the completion of (27) is indeed a completed tensor product of completions.

Putting the theorem together with Corollary 21 immediately yields the following. We say that two quantizations of $\mathcal{O}(X)$ are equivalent if they are isomorphic as $\mathbf{C} \llbracket \hbar \rrbracket$-algebras via a continuous isomorphism that is the identity modulo $\hbar$ (this is also known as gauge equivalence). In the $\mathbf{C}^{\times}$-compatible case, we can ask for a graded equivalence, i.e., an equivalence preserving the $\mathbf{C}^{\times}$-action.

Corollary 28. Let $X$ and $x$ be as in Corollary 21. Then the unique $\mathbf{C}^{\times}$-compatible quantization of $\hat{X}_{\mathbf{C}^{\times} \cdot x}$ up to graded equivalence is given in the theorem. In particular, every compatible quantization of $X$ restricts to this one, up to graded equivalence.

Proof of Theorem 26. This follows from the same argument as the proof in $\$ 2.1$ of Theorem 19, just like the case of uniqueness of the quantization of the formal disk $\Delta^{2 n}$ itself. Namely, let $Y$ be either $\mathbf{C}^{\times} \times \mathbf{A}^{2 n-1}$ or $\mathbf{C}^{\times} \times \Delta^{2 n-1}$. For any quantization $A_{\hbar}$ of $Y$, fix a $\mathbf{C} \llbracket \hbar \rrbracket$-module isomorphism $A_{\hbar} \cong \mathcal{O}(Y) \llbracket \hbar \rrbracket$, compatible with the projection to $\mathcal{O}(Y)$. We then can view the quantization as given by an associative, continuous multiplication $\star$ on $\mathcal{O}(Y) \llbracket \hbar \rrbracket$. 
Inductively, assume that, for $f, g \in \mathcal{O}(Y)$, then $f \star g-g \star f \equiv \hbar\{f, h\}\left(\bmod \hbar^{m}\right)$ for some $m \geq 2$. (It is clear, for the base case, that we can do this when $m=2$.) Then, as in the proof of Theorem 19, first we modify $z_{i}$ so that they commute with $t$ modulo $\hbar^{m}$. The reader can safely skip the remainder of the proof, since it closely follows $\$ 2.1$; we provide the details for completeness.

Let $[f, g]_{\star}:=f \star g-g \star f$. We iteratively apply the gauge transformation $z_{i} \mapsto z_{i}-$ $\hbar^{-1} \int\left[t, z_{i}\right]_{\star} d u$ (fixing the other coordinates $t, u$, and $z_{j}$ for $\left.j \neq i\right)$. If $\hbar^{-m}\left[t, z_{i}\right]_{\star}(\bmod \hbar)$ vanishes to order $k$ at the augmentation ideal $J=\left(u, z_{1}, \ldots, z_{2 n-2}\right)$, then the transformation is the identity modulo $\hbar^{m-1} J^{k+2}$ and, after applying it, $\hbar^{-m}\left[t, z_{i}\right]_{\star}(\bmod \hbar)$ vanishes to order $k+1$. So this sequence of transformations converges and, afterwards, $\left[t, z_{i}\right]_{\star} \equiv 0\left(\bmod \hbar^{m+1}\right)$, without affecting $\left[t, z_{j}\right]_{\star}$ for $j \neq i$ or any commutators modulo $\hbar^{2}$. Applying this to all the $z_{j}$ means that we can assume $\left[t, z_{j}\right]_{\star} \equiv 0\left(\bmod \hbar^{m+1}\right)$ for all $j$.

Let $B$ be the subalgebra of $\mathcal{O}(Y)$ which is generated by the $z_{i}$, i.e., $\mathbf{C}\left[z_{1}, \ldots, z_{2 n-2}\right]$ or $\mathbf{C} \llbracket z_{1}, \ldots, z_{2 n-2} \rrbracket$. As a consequence of the above, the degree-zero part of the centralizer of $t$ modulo $\hbar^{m+1}$ in $A_{\hbar} / \hbar^{m} A_{\hbar}$ under the star product is $B[\hbar] /\left(\hbar^{m}\right)$. Then, for each $i$, the vector field $\xi:=t^{k} \hbar^{-m}\left[z_{i},-\right]_{\star}(\bmod \hbar)$ is Poisson on $\mathbf{A}^{2 n-2}$ or $\Delta^{2 n-2}$ with respect to the Poisson backet $\left.\{-,-\}^{\prime}=t^{k}\{-,-\}\right)$ and hence Hamiltonian. Thus $\xi=t^{k}\{f,-\}$ for some $f \in B$ and we can perform a gauge transformation $z_{i} \mapsto z_{i}-\hbar^{m-1} f$ (fixing the other coordinates) so that $\left[z_{i}, z_{j}\right]_{\star} \equiv 0\left(\bmod \hbar^{m+1}\right)$ for all $j$. (Note that this is simpler than what we had to do in the Darboux theorem itself, owing to $m \geq 2$ ).

After all of this, the star-product commutators modulo $\hbar^{m+1}$ are as desired amongst the coordinates $t, z_{1}, \ldots, z_{2 n-2}$, and we are left only with commutators involving the coordinate $u$. Now, we have

$$
\left[t,\left[u, z_{i}\right]_{\star}\right]_{\star} \equiv\left[[t, u]_{\star}, z_{i}\right]_{\star} \equiv 0 \quad\left(\bmod \hbar^{m+2}\right)
$$

and hence $t^{k} \hbar^{-m}\left[u, z_{i}\right]_{\star}(\bmod \hbar)$ lies in $B$. Therefore, as before, $\xi:=t^{k} \hbar^{-m}[u,-]_{\star}(\bmod \hbar)$ is a Poisson and hence Hamiltonian vector field on $B$, so $\left.\xi\right|_{B}=t^{k}\{f,-\}$ for some $f \in B$. Then, after performing the gauge transformation $u \mapsto u-\hbar^{m-1} f$, we will obtain $\left[u, z_{i}\right]_{\star} \in \hbar^{m+1} A_{\hbar}$, as desired.

After all of these steps, we only need to make a further gauge transformation so that $[t, u] \equiv \hbar t^{k-1}\left(\bmod \hbar^{m+1}\right)$. Since $t$ and $u$ commute with the $z_{i}$ modulo $\hbar^{m+1}$, the centralizer of the $z_{i}$ modulo $\hbar^{m+1}$ is $\mathbf{C}\left[t, t^{-1}\right][u]$ or $\mathbf{C}\left[t, t^{-1}\right] \llbracket u \rrbracket$ (depending on whether we used $\mathbf{A}^{2 n-1}$ or $\left.\Delta^{2 n-1}\right)$. Since $[t, u]_{\star}$ has degree $1-k$, we obtain that $\hbar^{-m}\left(t^{k-1}[t, u]_{\star}-\hbar\right)(\bmod \hbar)$ is in $\mathbf{C}[u]$ or $\mathbf{C} \llbracket u \rrbracket$. We can therefore make the substitution $u \mapsto u-\hbar^{m} \int \hbar^{-m} t^{k-1}\left([t, u]_{\star}-\hbar\right) d u$, which will ensure that $[t, u] \equiv \hbar t^{1-k}\left(\bmod \hbar^{m+1}\right)$, and all brackets will be as desired modulo $\hbar^{m+1}$, completing the induction.

Remark 29. When $k>0$, one can instead ask about filtered quantizations. Namely, for $B$ a graded Poisson algebra with bracket of degree $-k$, one can ask for a filtered algebra $A=$ $\bigcup_{m} A_{\leq m}$ whose associated graded algebra $\operatorname{gr}(A)=\bigoplus_{m} \operatorname{gr}_{m}(A):=A_{\leq m} / A_{\leq m-1}$ is isomorphic to $B$ as an algebra, such that $\left[A_{\leq m}, A_{\leq n}\right] \subseteq A_{\leq m+n-k}$ and $\operatorname{gr}_{m+n-k}[a, b]=\left\{\operatorname{gr}_{m} a, \operatorname{gr}_{n} b\right\}$ for $a \in A_{\leq m}$ and $b \in A_{\leq n}$. If one asks also that $A$ be complete with respect to the descending part of the filtration, i.e., $A=\lim _{m \rightarrow-\infty} A / A_{\leq m}$ as a vector space (which is a trivial condition 
if $B$ is nonnegatively graded), then one can generalize the results above to show that there is a unique filtered quantization as well.

Note that, for $k=1$, this is a formal consequence, since then filtered quantizations are equivalent to $\mathbf{C}^{\times}$-compatible quantizations: to a filtered algebra we associate its completed Rees algebra; the reverse direction is given by taking $\mathbf{C}^{\times}$-finite vectors and then setting $\hbar=1$. But for $k \geq 2$, not all filtered quantizations can be obtained from $\mathbf{C}^{\times}$-compatible quantizations: for example, for $k=2$ and $B=\mathbf{C}[t, y]$ with $|y|=2,|t|=1$, and $\{t, y\}=t$, one can take the filtered quantization $\mathbf{C}\langle t, y\rangle /([t, y]-t-1)$, which cannot be obtained from a compatible quantization (but it can, in view of the uniqueness result above, after inverting $t$ and completing with respect to the filtration; then, in the above coordinates, $\left.y=\left(t^{2}+t\right) u\right)$. (One can, however, make a more direct link by working over $\mathbf{C} \llbracket \hbar^{1 / k} \rrbracket$ : then, every filtered quantization can be obtained from a $\mathbf{C} \llbracket \hbar^{1 / k} \rrbracket$-algebra with a compatible $\mathbf{C}^{\times}$action.)

Similarly, in all later results in this paper on quantization, one can obtain analogous results for filtered algebras by passing to the $\mathbf{C}^{\times}$-finite vectors and setting $\hbar=1$, in the case when $k$ is positive. We will not mention this further.

\section{Equivariant Darboux-Weinstein theorems}

In this section, we give the main theorems (34, 39, and 43), their application to $\mathcal{D}$-modules on Poisson varieties (Corollary 45 and Remark 47), and their quantization (Theorem 48). We begin in the first subsection with the statements of the main theorems and corollaries, and prove only the corollaries. We then give an application to $\mathcal{D}$-modules, followed by the proof of Theorem 1 and the main theorems, and finally discuss quantization. The proofs of the main theorems can be omitted on a first reading.

\subsection{Main results}

Recall that, in the non-equivariant setting, the formal Darboux-Weinstein theorem ([Wei83]; see also [Kal06, Proposition 3.3]) says that, for $Y \subseteq X$ an a symplectic leaf of a Poisson variety $X$ and $y \in Y$ a point, then the formal neighborhood $\hat{X}_{y}$ is Poisson isomorphic to a product, with $S$ a formal Poisson scheme (with a single closed point and Poisson bivector vanishing there):

$$
\hat{X}_{y} \cong \hat{Y}_{y} \times S \cong \Delta^{\operatorname{dim} Y} \times S .
$$

In the $\mathbf{C}^{\times}$-equivariant setting, this no longer holds when $y$ is not a fixed point, as in the following examples (although one can easily see it does hold for elliptic fixed points as in Remark 24).

Example 31. Consider $X=\mathbf{C}^{\times} \times \Delta^{2}=\operatorname{Spf} \mathbf{C}\left[t, t^{-1}\right] \llbracket u, z \rrbracket$ with Poisson bivector $\partial_{u} \wedge\left(t \partial_{t}+\partial_{z}\right)$ of degree zero (note that the resulting bracket automatically satisfies the Jacobi identity since the Poisson bivector has rank two). Then the Poisson center consists of those functions in $\mathbf{C}\left[t, t^{-1}\right] \llbracket z \rrbracket$ annihilated by the Hamiltonian vector field $\xi_{u}=t \partial_{t}+\partial_{z}$, i.e., $\mathbf{C}\left[t e^{-z}, t^{-1} e^{z}\right]$, whose spectrum is $\mathbf{C}^{\times}$with the dilating $\mathbf{C}^{\times}$action. On the other hand, the Poisson center 
of the product of the symplectic $\mathbf{C}^{\times}$-variety $\mathbf{C}^{\times} \times \Delta$ and $\Delta$ is the functions on the latter factor, $\mathbf{C} \llbracket z \rrbracket$, which is not isomorphic to $\mathbf{C}\left[t e^{-z}, t^{-1} e^{z}\right]$, and is equipped with the trivial $\mathbf{C}^{\times}$ action.

We remark that, to get a trivial Poisson center rather than $\mathbf{C}\left[t e^{-z}, t^{-1} e^{z}\right]$ as above, we could have instead used the Poisson bivector $\partial_{u} \wedge\left(t \partial_{t}+\gamma z \partial_{z}\right)$ for $\gamma \in \mathbf{C}$ irrational: then the Poisson center is trivial since the differential equation $\gamma z \partial_{z}(f)=m f$ has no solutions with $m \in \mathbf{Z}$ and $f \in \mathbf{C} \llbracket z \rrbracket$ (i.e., $z^{m / \gamma} \notin \mathbf{C} \llbracket z \rrbracket$ ).

Example 32. We can also give a singular example where the Poisson structure is nondegenerate on the smooth locus (in particular, this implies it is generically symplectic). Let $X=\mathbf{C}^{\times} \times \Delta \times Z$ where $Z$ is the formal neighborhood of the origin of the hypersurface $x^{3}+y^{3}+z^{3}=0$ in $\mathbf{C}^{3}$ (i.e., the cone over a smooth genus one curve in $\mathbf{C P}^{2}$ ). Let $\xi$ be the usual Euler vector field on $Z$, i.e., $x \partial_{x}+y \partial_{y}+z \partial_{z}$. Equip $Z$ with the Jacobian Poisson structure,

$$
\pi_{Z}=3\left(x^{2} \partial_{y} \wedge \partial_{z}+y^{2} \partial_{z} \wedge \partial_{x}+z^{2} \partial_{x} \wedge \partial_{y}\right)
$$

Now, $\xi$ is a Poisson vector field, i.e., $L_{\xi}\left(\pi_{Z}\right)=0$, where $L_{\xi}$ is the Lie derivative, or equivalently, $\left[\xi, \pi_{z}\right]=0$, where $[-,-]$ is the Schouten-Nijenhuis bracket. Therefore, the following bivector $\pi$ is Poisson (i.e., $[\pi, \pi]=0$ ):

$$
\pi=\partial_{u} \wedge\left(t \partial_{t}+\xi\right)+\pi_{Z}
$$

On the other hand, we claim that this is not equivalent via change of coordinates to a product of formal Poisson schemes $\left(\mathbf{C}^{\times} \times \Delta\right) \times Z$. Indeed, if it were, there would have to be a degree zero coordinate $u^{\prime}$ such that $\left\{u^{\prime}, t\right\}=t$ and $u^{\prime}$ commutes with $x, y$, and $z$. The first condition shows that $u^{\prime} \in u+f$ for some $f \in \mathbf{C} \llbracket x, y, z \rrbracket$, and the second condition shows that $\{f, g\}=-\xi(g)$ for all $g \in \mathbf{C} \llbracket x, y, z \rrbracket$. This means that $\xi$ is a Hamiltonian vector field on $\mathbf{C} \llbracket x, y, z \rrbracket$. But that is false, since every Hamiltonian vector field has positive degree, whereas $\xi$ has degree zero. This is a contradiction.

The preceding example demonstrates exactly what goes wrong, however, as we prove in the following theorem. Let $X$ be a $\mathbf{C}^{\times}$-Poisson variety with a homogeneous Poisson bivector, $Y \subseteq X$ be a $\mathbf{C}^{\times}$-invariant symplectic leaf, and $y \in Y$ be a point with minimal stabilizer under the $\mathbf{C}^{\times}$-action. If the stabilizer of $y$ is in fact trivial, then $\hat{Y}_{\mathbf{C}^{\times} \cdot y}$ has a standard symplectic structure (17). In general, we obtain a symplectic structure (23). Let $\hat{X}_{\mathbf{C}^{\times} \cdot y}$ denote the formal neighborhood of $\mathbf{C}^{\times} \cdot y$ in $X$ (equivalently, we can replace $X$ with any open affine subvariety in which the punctured line $\mathbf{C}^{\times} \cdot y$ is closed.) Let $t \in \mathcal{O}_{\hat{X}_{\mathbf{C}^{\times} \cdot y}}$ be the coordinate along the line $\mathbf{C}^{\times}$, i.e., $t(c \cdot y)=c$ for all $c \in \mathbf{C}^{\times}$.

For every (formal) scheme $Z$, let $\Omega_{Z}$ be the sheaf of Kähler differentials. If $Z^{\circ}$ is the smooth locus with inclusion $j: Z^{\circ} \rightarrow Z$, then we define $\tilde{\Omega}_{Z}:=j_{*} \Omega_{Z^{\circ}}$, the underived pushforward of $\Omega_{Z^{\circ}}$ (this differs from the definition of of [Fer70, Fer71] where one takes $\Omega_{Z}$ modulo torsion $(4)$. We will also use the complexes of de Rham differentials, $\Omega_{Z}^{\bullet}=\wedge_{\mathcal{O}_{X}} \Omega_{Z}$ and $\tilde{\Omega}_{Z}^{\bullet}:=j_{*} \Omega_{Z^{\circ}}$. Moreover, for us $Z$ will be affine, so $\Omega_{Z}$ and $\tilde{\Omega}_{Z}$ can be identified with

\footnotetext{
${ }^{4}$ Thanks to a referee for clarifying this point.
} 
their global sections. Assume that $Z$ is normal affine. Then, $H^{1}\left(\tilde{\Omega}_{Z}^{\bullet}\right)=H^{1} \Gamma\left(Z^{\circ}, \Omega_{Z^{\circ}}^{\bullet}\right.$, which equals global closed algebraic one-forms on $Z^{\circ} \bmod$ global exact algebraic one-forms. When $Z$ is a complex algebraic variety, this embeds into the topological de Rham cohomology $H^{1}\left(Z^{\circ}\right)$, because if an algebraic one-form $\alpha$ on a smooth variety is the differential of a $C^{\infty}$ function $f$, then $f$ must itself be algebraic. In particular, if $H^{1}\left(Z^{\circ}\right)=0$, then $H^{1}\left(\tilde{\Omega}_{Z}^{\bullet}\right)=0$.

For $Z$ Poisson, let $H(Z)$ denote the Lie algebra of Hamiltonian vector fields on $Z$, i.e., $H(Z)=\left\{\xi_{f} \mid f \in Z\right\}$, with $\xi_{f}(g):=\{f, g\}$. Let $P(Z)$ denote the Lie algebra of Poisson vector fields, i.e., vector fields $\xi$ which are Lie derivations of the Poisson bracket (equivalently, $[\xi, \pi]=0$, using the Schouten-Nijenhuis bracket, with $\pi$ the Poisson bivector). We always have $H(Z) \subseteq P(Z)$. When $Z$ is normal, affine, and symplectic on the smooth locus, then $P(Z) / H(Z) \cong H^{1}\left(\tilde{\Omega}_{Z}^{\bullet}\right)$, since for $\omega$ the generic symplectic form, then $i_{\xi} \omega$ is a regular oneform on $Z^{\circ}$ for every vector field $\xi$, and $\xi$ is Hamiltonian or Poisson if and only if $i_{\xi} \omega$ is closed or exact, respectively.

Theorem 34. Assume that $y$ has trivial stabilizer under $\mathbf{C}^{\times}$. In a formal neighborhood of $\mathbf{C}^{\times} \cdot y$, with all isomorphisms $\mathbf{C}^{\times}$-equivariant,

(i) $\hat{Y}_{\mathbf{C}^{\times} \cdot y} \cong \mathbf{C}^{\times} \times \Delta^{\operatorname{dim} Y-1}$ with a standard homogeneous symplectic structure (17) of degree $k$.

(ii) Forgetting the Poisson structure,

$$
\hat{X}_{\mathbf{C}^{\times} \cdot y} \cong \hat{Y}_{\mathbf{C}^{\times} \cdot y} \times S,
$$

for some formal scheme $S$ with the trivial $\mathbf{C}^{\times}$action. The projection $\hat{X}_{\mathbf{C}^{\times} \cdot y} \rightarrow \hat{Y}_{\mathbf{C}^{\times} \cdot y}$ is Poisson. The second projection is not Poisson, but $t^{k}\{-,-\}$ restricts on $\mathcal{O}(S)$ to a Poisson bracket.

(iii) We can choose coordinates as above so that the Poisson bivector on $\hat{X}_{\mathbf{C}^{\times} \cdot y}$ has the form

$$
\pi_{\operatorname{dim} Y,-k}+t^{-k} \partial_{u} \wedge \xi+t^{-k} \pi_{S}
$$

for $\pi_{S}$ the Poisson bivector on $S$, and $\xi$ a vector field on $S$ satisfying $\left[\xi, \pi_{S}\right]=k \pi_{S}$.

More generally, in the case where the stabilizer of $y$ is arbitary, we deduce:

Corollary 37. (i) $\hat{Y}_{\mathbf{C}^{\times} \cdot y} \cong \mathbf{C}^{\times} \times \Delta^{\operatorname{dim} Y-1}$ with a standard homogeneous symplectic structure (23) of degree $k$.

(ii) Let $\tilde{\hat{Y}}_{\mathbf{C}^{\times} \cdot y} \rightarrow \hat{Y}_{\mathbf{C}^{\times} \cdot y}$ be the $\ell$-fold étale cover obtained by adjoining $t^{1 / \ell}$. Then, there is a canonical formal Poisson scheme $S$ with a $\mathbf{Z} / \ell$ action and a continuous $\mathbf{C}^{\times}$-equivariant isomorphism

$$
\hat{X}_{\mathbf{C}^{\times} \cdot y} \cong\left(\tilde{\hat{Y}}_{\mathbf{C}^{\times} \cdot y} \times S\right) /(\mathbf{Z} / \ell),
$$

which is Poisson when the RHS is equipped with a structure as in (36). 
Proof. Part (i) is an immediate consequence of Corollary 21,

Part (ii) follows directly from Theorem 34. Namely, when we adjoin $t^{1 / \ell}$, then the latter coordinate again has degree 1 , so we can apply the theorem and get a product decomposition for the étale $\ell$-fold cover.

For simplicity, we restrict for the remainder of the subsection to the case $\ell=1$. To avoid confusion, when $S$ is a formal Poisson scheme, we let the Hamiltonian vector fields of functions on $S$ be denoted as $\xi_{f}^{S}$.

Theorem 39. Let $m \geq 1$ and fix $k \in \mathbf{Z}$. Given two formal Poisson schemes $S$, $S^{\prime}$ with Poisson bivectors $\pi_{S}$ and $\pi_{S^{\prime}}$, equipped with vector fields $\xi, \xi^{\prime}$ satisfying $\left[\xi, \pi_{S}\right]=k \pi_{S}$ and $\left[\xi^{\prime}, \pi_{S^{\prime}}\right]=k \pi_{S^{\prime}}$, the formal Poisson schemes $\mathbf{C}^{\times} \times \Delta^{2 m-1} \times S$ and $\mathbf{C}^{\times} \times \Delta^{2 m-1} \times S^{\prime}$ with bivectors (36) are $\mathbf{C}^{\times}$-equivariantly Poisson isomorphic (with $\mathbf{C}^{\times}$acting only on the first factor) if and only if there is formal Poisson isomorphism $S \rightarrow S^{\prime}$ taking $\xi$ to $\xi^{\prime}+\xi_{f}^{S}$ for some $f \in \mathcal{O}_{S^{\prime}}$.

Remark 40. As we will see in the proof, the isomorphisms in the theorem can be chosen to form a commutative square:

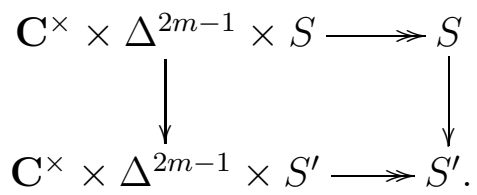

Corollary 41. If $S$ has the property that all Poisson vector fields are Hamiltonian, then all Poisson bivectors of the form (36) (i.e., for all choices of $\xi$ satisfying $\left[\xi, \pi_{S}\right]=k \pi_{S}$ ) are $\mathrm{C}^{\times}$-Poisson isomorphic.

Proof. If $\xi$ and $\xi^{\prime}$ satisfy $\left[\xi, \pi_{S}\right]=k \pi_{S}=\left[\xi^{\prime}, \pi_{S}\right]$, then $\xi-\xi^{\prime}$ is Poisson. Under the assumption of the corollary, it is also Hamiltonian. Then, by Theorem 39, taking $S=S^{\prime}$ and the identity map $S \rightarrow S^{\prime}=S$, we conclude that the two Poisson bivectors (36) given by $\xi$ and $\xi^{\prime}$ are related by a $\mathbf{C}^{\times}$-Poisson automorphism. (By the remark, it can be chosen to be compatible with the projection to $S$.)

Corollary 42. For a fixed formal Poisson scheme $S$ and fixed $m \geq 1$ and $k \in \mathbf{Z}$ equipped with a vector field $\eta$ satisfying $\left[\eta, \pi_{S}\right]=k \pi_{S}$, the set of $\mathbf{C}^{\times}$-Poisson isomorphism classes of bivectors of the form (36) is in bijection with $(\eta+P(S) / H(S)) / \operatorname{Aut}\left(S, \pi_{S}\right)$.

Note that, if $S$ is normal and symplectic on the smooth locus, we can alternatively write $(\eta+P(S) / H(S)) / \operatorname{Aut}\left(S, \pi_{S}\right)$ as $\left(i_{\xi} \omega+H^{1}\left(\tilde{\Omega}_{S}^{\bullet}\right)\right) / \operatorname{Aut}\left(S, \pi_{S}\right)$.

Proof of Corollary 42. Let $\xi$ be any other vector field on $S$ satisfying $\left[\xi, \pi_{S}\right]=k \pi_{S}$. The theorem implies that the resulting bivectors given by (36) are isomorphic if and only if $\xi \in \operatorname{Aut}\left(S, \pi_{S}\right) \cdot(\eta+H(S))$. On the other hand, $\xi-\eta \in P(S)$, so $\xi \in(\eta+P(S)) \subseteq \operatorname{Aut}\left(S, \pi_{S}\right)$. $(\eta+P(S))$, proving the result (note that this inclusion $\subseteq$ is actually an equality). 
Next we explain why, when $X$ admits a symplectic resolution, all bivectors in (36) are equivalent. This is similar to [PS16, Proposition 5.5]:

Theorem 43. Let $X$ be a normal Poisson variety, $Y$ a symplectic leaf, and $y \in Y$. Let $S$ be a formal Poisson scheme appearing either in an ordinary Darboux-Weinstein decomposition $\hat{X}_{y} \cong \hat{Y}_{y} \times S$, or in an equivariant one $\hat{X}_{\mathbf{C}^{\times} \cdot y} \cong \hat{Y}_{\mathbf{C}^{\times} \cdot y} \times S$ of Theorem 34 for $y$ having trivial stabilizer in $\mathbf{C}^{\times}$. If $X$ admits a symplectic resolution, then all Poisson vector fields on $S$ are Hamiltonian. More generally, each of the following conditions implies the next:

(i) $X$ admits a symplectic resolution, or more generally is a symplectic singularity;

(ii) $X$ is generically symplectic and there is an analytic neighborhood $U$ of $y$ such that, for $U^{\circ}$ the smooth locus of $U$, we have $H^{1}\left(U^{\circ}, \mathbf{C}\right)=0$;

(iii) $X$ is generically symplectic and $H^{1}\left(\tilde{\Omega}_{S}^{\bullet}\right)=0$; and

(iv) All Poisson vector fields on $S$ are Hamiltonian.

In the presence of a $\mathbf{C}^{\times}$-action on $S$, we can put the above results together to conclude:

Corollary 44. Let $X, Y, y, d$ be as in Theorem 34 and suppose that the stabilizer of $y$ is trivial. For a decomposition therein, suppose that $S$ admits a $\mathbf{C}^{\times}$action such that the Poisson structure is homogeneous of degree $-k$, and that all Poisson vector fields on $S$ are Hamiltonian (e.g., if $X$ admits a symplectic resolution or is a symplectic singularity). Then $\hat{X}_{\mathbf{C}^{\times} \cdot y}$ is $\mathbf{C}^{\times}$-equivariantly Poisson isomorphic to $\left(\hat{Y}_{\mathbf{C}^{\times} \cdot y} \times S, \pi_{\hat{Y}_{\mathbf{C}^{\times} \cdot y}}+\pi_{S}\right)$, with the new action of $\mathbf{C}^{\times}$on $S$. Moreover, in this case, $S$ is isomorphic to every slice appearing in an ordinary Darboux-Weinstein decomposition $\hat{X}_{y} \cong \hat{Y}_{y} \times S$.

Proof. Let $\eta$ be the Euler vector field of the action of $\mathbf{C}^{\times}$on $S$. Then $\left[\eta, \pi_{S}\right]=-k \pi_{S}$. Therefore, by Theorem 39, we can assume $\xi=-\eta$ in (36). Let $B \subseteq \mathcal{O}_{S}\left[t, t^{-1}\right]$ be the subalgebra of elements of weight zero with respect to the total grading by $|t|=1$ and the weight grading on $\mathcal{O}_{S}$ produced by the $\mathbf{C}^{\times}$-action. That is, $B$ consists of the elements of $f \in \mathcal{O}_{S}\left[t, t^{-1}\right]$ annihilated by $-\eta+t \partial_{t}=\xi+t \partial_{t}$. In other words, $B$ is the subalgebra of $\mathcal{O}_{S}\left[t, t^{-1}\right]$ of elements commuting with $u$. We therefore obtain $X=\left(\mathbf{C}^{\times} \times \Delta^{\operatorname{dim} Y-1}\right) \times \operatorname{Spf} B$, where now this is a product of formal schemes, with the Poisson structure on the first factor $\mathbf{C}^{\times} \times \Delta^{\operatorname{dim} Y-1}$ the standard one of (17). The first statement then follows from Theorem 19, For the second statement, we can complete at $y$ and obtain an ordinary Darboux-Weinstein decomposition. But, the $S$ appearing in this decomposition is unique up to formal Poisson automorphisms, since $S$ is the centralizer of $\hat{\mathcal{O}}_{Y, y}$, and two choices of subalgebra $\hat{\mathcal{O}}_{Y, y} \subseteq \hat{\mathcal{O}}_{X, y}$, i.e., of projections $\hat{X}_{y} \rightarrow \hat{Y}_{y}$, differ by Hamiltonian isomorphisms (cf. the proof of Theorem 39 below).

\subsection{Consequence for the $\mathcal{D}$-module on $X$}

Recall from [ES10] the $\mathcal{D}$-module, $M(X)$, on $X$ which represents solutions of Hamiltonian flow. For simplicity, assume $X$ is affine. Then this is defined by $M(X)=H(X) \mathcal{D}_{X} \backslash \mathcal{D}_{X}$, for 
$H(X)$ the Lie algebra of Hamiltonian vector fields on $X, \mathcal{D}_{X}$ the canonical right $\mathcal{D}$-module on $X$ such that $\operatorname{Hom}\left(\mathcal{D}_{X},-\right)$ is the global sections functor (i.e., $\mathcal{D}_{X}=\iota^{!}\left(I_{X} \mathcal{D}_{V} \backslash \mathcal{D}_{V}\right)$ where $\iota: X \rightarrow V$ is any embedding into a smooth affine variety $V$ with $X$ having ideal $\left.I_{X}\right)$, and with $H(X)$ acting on $\mathcal{D}_{X}$ on the left via the inclusion $H(X) \hookrightarrow \Gamma\left(\mathcal{D}_{X}\right)=$ differential operators on $X$. See [ES10] for more details.

We may deduce from the preceding the following consequence on the $\mathcal{D}$-module $M(X)$. Let $i: Y \rightarrow X$ be the inclusion of a symplectic leaf $Y$ into $X$.

Corollary 45. Suppose that $X$ is a symplectic singularity and that the assumptions of Corollary 44 are satisfied. Then, as weakly equivariant $\mathcal{D}$-modules on $\hat{Y}_{\mathbf{C}^{\times} \cdot y}$,

$$
\left.H^{0}\left(i^{*} M(X)\right)\right|_{\hat{Y}_{\mathbf{C}^{\times} \cdot y}} \cong \Omega_{\hat{Y}_{\mathbf{C}^{\times} \cdot y}} \otimes \operatorname{HP}_{0}(S),
$$

equipping $\operatorname{HP}_{0}(S)$ with the weight grading by the $\mathbf{C}^{\times}$-action on $S$.

Proof. First, it is easy to see that $\left.H^{0}\left(i^{*} M(X)\right)\right|_{\hat{Y}_{\mathbf{C}^{\times} \cdot y}}$ is a local system on $\hat{Y}_{\mathbf{C}^{\times} \cdot y}$ (as noticed in [ES10, §4.3], this is already true restricting to $Y$ itself; we use here that $X$ is a union of finitely many symplectic leaves, which is true for every symplectic singularity). As noticed there, the fibers of this local system are identified with $\mathrm{HP}_{0}\left(\mathcal{O}_{S}\right)$ (which is finite-dimensional). Now, in terms of the decomposition of Corollary 44, everything is weakly $\mathbf{C}^{\times}$-equivariant, equipping $\operatorname{HP}_{0}\left(\mathcal{O}_{S}\right)$ with its weight grading.

Remark 47. The assumptions of Corollary 44 can be dropped, as follows. All weakly $\mathbf{C}^{\times}$equivariant local systems on a formal neighborhood of a punctured line $\mathbf{C}^{\times} \cdot y$ in a smooth variety are sums of weight-shifted trivial local systems. Therefore, if we assume nothing but the fact that $Y$ is a symplectic leaf closed under the $\mathbf{C}^{\times}$-action and that $X$ is a symplectic singularity, we know that the LHS of (46) must be a weakly equivariant local system on $\mathbf{C}^{\times} \cdot y$ (at this point, we only need the symplectic singularity condition to guarantee that $M(X)$ is holonomic, which more generally follows if $X$ is a union of finitely many symplectic leaves). We can describe this local system using an ordinary Darboux-Weinstein slice $S$, i.e., such that $\hat{X}_{y} \cong \hat{Y}_{y} \times S$. Then, similarly to [PS16, $\left.\$ 5\right]$, the grading on $\operatorname{HP}_{0}(S)$ is given by an arbitrary vector field $\eta$ such that $\left[\eta, \pi_{S}\right]=-k \pi_{S}$ (using now the fact that all Poisson vector fields are Hamiltonian, from Theorem 433). Such a vector field can be obtained from the Euler vector field $\mathrm{Eu}_{X}$ of $X$ by the projection $\pi_{S}: \hat{X}_{y} \rightarrow S$, namely $\eta=\left(\pi_{S}\right)_{*}\left(\left.\mathrm{Eu}_{X}\right|_{\{0\} \times S}\right)$.

\subsection{Proof of Theorem 1}

Here we deduce Theorem 1 from the preceding theorems. Part (i) is an immediate consequence of Theorem 34.(i). Part (ii) is an immediate consequence of Theorem 19 and Theorem 34,(ii). Part (iii) follows from Corollary 41 and Theorem 43, Part (iv) is identical to Corollary 44. 


\subsection{Proof of Theorem 34}

We first point out that, if the reader has not seen the proof in the simpler symplectic setting in 92.1 , it may be helpful to read that first, as the proof of (ii) below follows the same ideas but is a bit more complicated.

Part (i) of Theorem 34 is an immediate consequence of Corollary 16, together with the fact that the formal neighborhood of any point in a smooth irreducible variety of dimension $n$ is isomorphic to $\Delta^{n}$.

Part (ii) is the main part of the proof. We will apply Kal06, Lemma 3.2], following the proof of [Kal06, Proposition 3.3]. Write $\hat{Y}_{\mathbf{C}^{\times} \cdot y}=\left(\mathbf{C}^{\times} \times \Delta\right) \times \Delta^{\operatorname{dim} Y-2}$ with its standard symplectic structure (17). Let $t, u$ denote homogeneous lifts to $\hat{X}_{\mathbf{C}^{\times} \cdot y}$ of the corresponding coordinate functions on $\hat{Y}_{\mathbf{C}^{\times} \cdot y}$ (we actually only require that $t$ is invertible of weight one and $\{t, u\}(y)=1$, so not the full strength of Theorem [19). Let $Z:=\operatorname{Spf} \mathbf{C}\left[t, t^{-1}\right] \llbracket u \rrbracket$, so that we have the projection $X \rightarrow Z$ given by the inclusion $\mathbf{C}\left[t, t^{-1}\right] \llbracket u \rrbracket$.

Consider the subalgebra $A \subseteq \hat{\mathcal{O}}_{X, \mathbf{C}^{\times} \cdot y}$ of degree-zero elements commuting with $t$. Let $X^{\prime}:=\operatorname{Spf} A$, so that there is a projection $X \rightarrow X^{\prime}$. Note that, although $A$ is not a Poisson subalgebra, $A\left[t, t^{-1}\right]$ is, since it consists of the $\mathbf{C}^{\times}$-locally finite elements which commute with $t$. Since $\{-,-\}$ has degree $-k$, we conclude that $\{-,-\}^{\prime}:=t^{k}\{-,-\}$ restricts to a Poisson bracket on $X^{\prime}$.

We claim that, as $\mathbf{C}^{\times}$-formal schemes, $\hat{X}_{\mathbf{C}^{\times} \cdot y} \cong Z \times X^{\prime}$ using the two projections. For this we apply [Kal06, Lemma 3.2], following the argument of [Kal06, Proposition 3.3]. View $\hat{\mathcal{O}}_{X, \mathbf{C}^{\times} \cdot y}^{\mathbf{C}^{\times}}$as a $\mathbf{C} \llbracket u \rrbracket=\mathcal{O}_{\Delta}$-module. Let $J \subseteq \hat{\mathcal{O}}_{X, \mathbf{C}^{\times} \cdot y}^{\mathbf{C}^{\times}}$be the degree-zero part of the ideal in $\hat{\mathcal{O}}_{X, \mathbf{C}^{\times} \cdot y}$ of $\mathbf{C}^{\times} \cdot y$; note that $J$ defines the topology on $\mathcal{O}_{X, \mathbf{C}^{\times} \cdot y}^{\mathbf{C}^{\times}}$. Consider the filtration $J^{\bullet}$ on $\mathcal{O}_{X, \mathbf{C}^{\times} \cdot y}^{\mathbf{C}^{\times}}$given by powers of $J$. Note that $J^{m}$ has finite codimension for all $m$ (since $J$ is a defining ideal for the topology on the affine formal scheme $\mathcal{O}_{X, \mathbf{C}^{\times} \cdot y}^{\mathbf{C}^{\times}}$which has a unique closed point, or this can be seen directly). Note also that $u J^{m} \subseteq J^{m+1}$ for all $m$. Note that $t^{k-1}\{t, u\} \equiv 1(\bmod J)$, hence $\{t, u\}$ is invertible. Consider the operator $\nabla_{u}$ defined by $\nabla_{u}(f):=\{t, u\}^{-1}\{t, f\}$. This satisfies $\left[\nabla_{u}, u\right]=0$, hence defines a flat connection on $\hat{\mathcal{O}}_{X, \mathbf{C}^{\times} \cdot y}^{\mathbf{C}^{\times}}$as a $\mathbf{C} \llbracket u \rrbracket$-module. Therefore the assumptions of [Kal06, Lemma 3.2] are satisfied, and the lemma yields that $\hat{\mathcal{O}}_{X, \mathbf{C}^{\times} \cdot y}^{\mathbf{C}^{\times}} \cong \mathbf{C} \llbracket u \rrbracket \hat{\otimes}\left(\hat{\mathcal{O}}_{X, \mathbf{C}^{\times} \cdot y}^{\mathbf{C}^{\times}}\right)^{\nabla}$. Note that the second factor is the subalgebra of degree-zero elements commuting with $t$. Since $\hat{\mathcal{O}}_{X, \mathbf{C}^{\times} \cdot y}$ is the completion of $\hat{\mathcal{O}}_{X, \mathbf{C}^{\times} \cdot y}^{\mathbf{C}^{\times}}\left[t, t^{-1}\right]$, we obtain also that

$$
\hat{\mathcal{O}}_{X, \mathbf{C}^{\times} \cdot y} \cong \mathbf{C}\left[t, t^{-1}\right] \llbracket u \rrbracket \hat{\otimes}\left(\left(\hat{\mathcal{O}}_{X, \mathbf{C}^{\times} \cdot y}\right)^{\nabla}\right)^{\mathbf{C}^{\times}},
$$

i.e., $\hat{X}_{\mathbf{C}^{\times} \cdot y} \cong Z \times X^{\prime}$ with the projections as claimed.

Next, applying the formal Darboux-Weinstein theorem to $X^{\prime}$, we obtain a Poisson isomorphism $X^{\prime} \cong \Delta^{\operatorname{dim} Y-2} \times S$ for some formal Poisson scheme $S$. Therefore, we get a decomposition of $\mathbf{C}^{\times}$-formal schemes $\hat{X}_{\mathbf{C}^{\times} \cdot y} \cong Z \times \Delta^{\operatorname{dim} Y-2} \times S$, which is not Poisson, but for which $t^{k}\{-,-\}$ restricts to a Poisson bracket on $\mathcal{O}(S)$ (as well as to the standard symplectic structure on $\left.\mathcal{O}\left(\Delta^{\operatorname{dim} Y-2}\right)\right)$.

However, it is unfortunately not quite true that the projection $X_{\mathbf{C}^{\times} \cdot y} \rightarrow Z \times \Delta^{\operatorname{dim} Y-2}$ is Poisson. In terms of the standard coordinates $t, u, z_{1}, \ldots, z_{\operatorname{dim} Y-2}$, the Poisson brackets are 
all those of the standard symplectic structure (17) except for those of $u$. To fix this, we need to change the coordinate $u$ so that $\left\{u, z_{i}^{\prime}\right\}=0$ for all $i$, with $z_{i}^{\prime}$ defined just after (17), and also so that $\{t, u\}=t^{1-k}$.

We explicitly construct in this paragraph a change of coordinates $u \mapsto u^{\prime}$ (fixing the other coordinates) so that $\left\{u^{\prime}, z_{i}^{\prime}\right\}=0$ for all $i$; an alternative (but nonexplicit) construction using [Kal06, Lemma 3.2] is given in the next paragraph. Let us use the notation $\int f d z_{j}$ for the antiderivative of $f \in \mathbf{C}\left[t, t^{-1}\right] \llbracket u, z_{1}, \ldots, z_{\operatorname{dim} Y-2} \rrbracket$ which is a multiple of $z_{j}$, which we extend $\mathcal{O}(S)$-linearly to $\hat{\mathcal{O}}_{X, \mathbf{C}^{\times} \cdot y}$. Let $\tilde{J}$ be the ideal of $\mathbf{C}^{\times} \cdot y$, i.e., $\tilde{J}$ is generated by $u, z_{1}, \ldots, z_{\operatorname{dim} Y-2}$, and the augmentation ideal of $\mathcal{O}(S)$. We then have that $\left\{u, z_{i}^{\prime}\right\} \in \tilde{J}$ for all $i$ by construction (since it is homogeneous and $\left\{u, z_{i}^{\prime}\right\}(y)=0$ ). Suppose inductively that $\left\{u, z_{i}^{\prime}\right\} \in(\tilde{J})^{m}$ for some $m \geq 1$. Then if $i$ is odd, we can make the change of coordinates $u \mapsto u^{\prime}:=$ $u+\int\left\{u, z_{i}^{\prime}\right\} d z_{i+1}$, which is congruent to $u$ modulo $(\tilde{J})^{m+2}$, and satisfies $\left\{u^{\prime}, z_{i}^{\prime}\right\} \in(\tilde{J})^{m+1}$. Moreover, if we had in fact $\left\{u, z_{j}^{\prime}\right\}=0$ for some $j$, then we claim that still $\left\{u^{\prime}, z_{j}^{\prime}\right\}=0$ :

$$
\left\{u^{\prime}, z_{j}^{\prime}\right\}=\left\{\int\left\{u, z_{i}^{\prime}\right\} d z_{i+1}, z_{j}^{\prime}\right\}=\int\left\{u, z_{j}^{\prime}\right\} d z_{i+1}=0,
$$

where for the second equality we use that $\left\{\int f d z_{i+1}, z_{j}^{\prime}\right\}=\int\left\{f, z_{j}^{\prime}\right\} d z_{i+1}$ for all $f$, which follows since $\left\{z_{i+1}, z_{j}^{\prime}\right\}$ is a power of $t$. Thus, iteratively performing the change of coordinates $u \mapsto u^{\prime}$ we get a convergent change of coordinates after which $u$ commutes with $z_{i}^{\prime}$ without affecting the property of commuting with other $z_{j}^{\prime}$. We can do the same procedure as above if $i$ is even, except making the change of coordinates $u \mapsto u^{\prime}:=u-t^{k} \int\left\{u, z_{i}^{\prime}\right\} d z_{i-1}$ (note $z_{i}^{\prime}=z_{i}$ in this case). Thus we have explicitly shown how to change coordinates so that $u$ commutes with $z_{i}^{\prime}$ for all $i$.

Alternatively, we could have again appealed to [Kal06, Lemma 3.2]: consider again $\hat{\mathcal{O}}_{X, \mathbf{C}^{\times} \cdot y}^{\mathbf{C}^{\times}}$filtered by powers of $J$, viewed now as a $\mathbf{C} \llbracket z_{1}, \ldots, z_{\operatorname{dim} Y-2} \rrbracket$-module. Define the operators $\nabla_{z_{i}}:=\left\{z_{i}^{\prime},-\right\}$ for $i$ odd and $\nabla_{z_{i}}:=t^{k}\left\{z_{i},-\right\}=t^{k}\left\{z_{i}^{\prime},-\right\}$ for $i$ even. This defines a connection since $\nabla_{z_{i}}\left(z_{j} f\right)=z_{j} \nabla_{z_{i}}(f)+\delta_{i j} f$ and it is flat since the $\nabla_{z_{i}}$ commute. Therefore [Kal06, Lemma 3.2] implies that $\hat{\mathcal{O}}_{X, \mathbf{C}^{\times} \cdot y}^{\mathbf{C}^{\times}} \cong \mathbf{C} \llbracket z_{1}, \ldots, z_{\operatorname{dim} Y-2} \rrbracket \hat{\otimes}\left(\hat{\mathcal{O}}_{X, \mathbf{C}^{\times} \cdot y}^{\mathbf{C}^{\times}}\right) \nabla$, and the latter factor is the centralizer of the $z_{i}^{\prime}$. There must be an element of the latter factor, call it $u^{\prime}$, such that $\left\{t, u^{\prime}\right\}$ does not vanish along $\mathbf{C}^{\times} \cdot y$ (as there is no such element in the first factor). The change of coordinates $u \mapsto u^{\prime}$ will then have the desired property.

Finally, we change coordinates so that $\{t, u\}=t^{1-k}$, using an explicit algorithm similar to the case $\left\{u, z_{i}^{\prime}\right\}=0$. Since $u$ and $t$ now commute with $z_{i}^{\prime}$ for all $i$, so must $\{t, u\}$, so that

$$
\{t, u\} \in t^{1-k}\left(1+(\mathbf{C} \llbracket u \rrbracket \hat{\otimes} \mathcal{O}(S))_{+}\right),
$$

with the subscript of + denoting the augmentation ideal. Suppose inductively that $\{t, u\}-$ $t^{1-k} \in\left(\mathbf{C} \llbracket u \rrbracket \hat{\otimes} \mathcal{O}(S)_{+}\right)^{m}$ for some $m \geq 1$ (which is guaranteed for $m=1$ ). Then, we perform the change of coordinates $u \mapsto u^{\prime}=u+\int t^{k-1}(1-\{t, u\}) d u$, which will have the property that $\left\{t, u^{\prime}\right\}-t^{1-k} \in\left(\mathbf{C} \llbracket u \rrbracket \hat{\otimes} \mathcal{O}(S)_{+}\right)^{m+1}$, completing the induction step. Note that these changes of coordinates will not affect the property $\left\{u, z_{i}^{\prime}\right\}=0$.

After this, we have that the projection $\hat{X}_{\mathbf{C}^{\times} \cdot y} \rightarrow Z \times \Delta^{\operatorname{dim} Y-2}$ is Poisson, as desired. To conclude, note that the composition $\hat{Y}_{\mathbf{C}^{\times} \cdot y} \rightarrow \hat{X}_{\mathbf{C}^{\times} \cdot y} \rightarrow Z \times \Delta^{\operatorname{dim} Y-2}$ of the inclusion with the 
projection to the first two factors is $\mathbf{C}^{\times}$-equivariant, sends $y$ to $((1,0), 0) \in Z \times \Delta^{\operatorname{dim} Y-2}$ and is an isomorphism on the tangent space. By equivariance, it is an isomorphism at the tangent spaces of all closed points and a bijection of closed points, hence it is an isomorphism. As it is a composition of Poisson morphisms, this isomorphism is Poisson. We can therefore compose with the inverse of this isomorphism to view our decomposition as $\hat{X}_{\mathbf{C}^{\times} \cdot y} \cong \hat{Y}_{\mathbf{C}^{\times} \cdot y} \times S$, with the first projection Poisson. Finally, the Poisson structure $\{-,-\}^{\prime}=t^{k}\{-,-\}$ on $X^{\prime}$ induces a Poisson structure on $S$ via the projection $X^{\prime} \rightarrow S$, also given by the same formula.

We now prove (iii). By part (ii), we can write the Poisson bivector on $\hat{X}_{\mathbf{C}^{\times} \cdot y} \cong \hat{Y}_{\mathbf{C}^{\times} \cdot y} \times S$ as

$$
\pi_{\hat{X}_{\mathbf{C}^{\times} \cdot y}}=\pi_{\hat{Y}_{\mathbf{C}^{\times} \cdot y}}+t^{k} \pi_{S}+\pi^{\prime}
$$

where $\pi^{\prime}$ is a bivector which projects to zero on each factor. As in (ii), set $Z=\mathbf{C}^{\times} \times \Delta$ and write $\hat{Y}_{\mathbf{C}^{\times} \cdot y} \cong Z \times \Delta^{\operatorname{dim} Y-2}$ with the standard symplectic structure (17). By construction, $\{t, \mathcal{O}(S)\}$ and $\left\{\mathcal{O}\left(\Delta^{\operatorname{dim} Y-2}\right), \mathcal{O}(S)\right\}$ are zero. Therefore $\pi^{\prime}$ has the form $\pi^{\prime}=t^{-k} \partial_{u} \wedge \xi$ where $\xi\left(\hat{\mathcal{O}}_{Y, \mathbf{C}^{\times} \cdot y}\right)=0$, that is, $\xi$ is constant in the $\hat{Y}_{\mathbf{C}^{\times} \cdot y}$ direction, and $\xi$ has degree zero. To conclude, it suffices to show that $\xi$ is actually parallel to $S$, i.e., $\xi(\mathcal{O}(S)) \subseteq \mathcal{O}(S)$. Note that $\xi=t^{k} \xi_{u}$ where $\xi_{u}$ is the Hamiltonian vector field of $u$. But, $\mathcal{O}(S)$ is the subalgebra of degree-zero elements commuting with the coordinate functions $t, z_{1}, \ldots, z_{\operatorname{dim} Y-2}$ on $\hat{Y}_{\mathbf{C}^{\times} \cdot y}$. To show this is preserved by $\xi$, it suffices to show that the subalgebra $\mathbf{C}\left[t, t^{-1}\right] \hat{\otimes} \mathcal{O}(S)$ of all elements commuting with $t, z_{1}, \ldots, z_{\operatorname{dim} Y-2}$ is preserved by $\xi_{u}$. This follows since $\xi_{t}(f)=$ $0=\xi_{z_{i}}(f)=0$ for all $i$, then $\xi_{t}\left(\xi_{u}(f)\right)=\left[\xi_{t}, \xi_{u}\right](f)=\xi_{\{t, u\}}(f)=\xi_{t^{-k-1}}(f)=0$ and $\xi_{z_{i}}\left(\xi_{u}(f)\right)=\left[\xi_{z_{i}}, \xi_{u}\right](f)=\xi_{\left\{z_{i}, u\right\}}(f)$, which again is zero since $\left\{z_{i}, u\right\}$ is either zero (if $i$ is even) or $-k t^{-k} z_{i}$ (if $i$ is odd). Thus, we obtain (36) with $\xi$ a vector field on $S$.

It remains to verify that $\left[\xi, \pi_{S}\right]=k \pi_{S}$. Since $\xi$ is Hamiltonian on $\hat{X}_{\mathbf{C}^{\times} \cdot y}$, it follows that $[\xi, \pi]=0$ where $\pi$ is the Poisson bivector on $\hat{X}_{\mathbf{C}^{\times} \cdot y}$. Since $\pi_{S}$ is the restriction of $t^{k} \pi$ to $S$ and $\xi$ is parallel to $S$, we obtain $\left[\xi, \pi_{S}\right]=\left.\xi\left(t^{k}\right) \pi\right|_{S}=\left.k t^{k} \pi\right|_{S}=k \pi_{S}$.

\subsection{Proof of Theorem 39}

Fix $m$ and $k$, and suppose we are given $\left(S, \pi_{S}, \xi\right)$ and $\left(S^{\prime}, \pi_{S^{\prime}}, \xi^{\prime}\right)$ satisfying $\left[\xi, \pi_{S}\right]=k \pi_{S}$ and $\left[\xi^{\prime}, \pi_{S^{\prime}}\right]=k \pi_{S^{\prime}}$. Suppose that we have a $\mathbf{C}^{\times}$-Poisson isomorphism

$$
\Phi: \mathbf{C}^{\times} \times \Delta^{2 m-1} \times S \rightarrow \mathbf{C}^{\times} \times \Delta^{2 m-1} \times S^{\prime},
$$

equipping both with the Poisson bivectors (36) and the $\mathbf{C}^{\times}$-actions by acting on the first factor. Let $t, u, z_{i}$ and $t^{\prime}, u^{\prime}, z_{i}^{\prime}$ be the corresponding coordinates on each satisfying $\left\{z_{2 i-1}, z_{2 i}\right\}=$ 1 (so, the coordinates which were denoted $z_{i}^{\prime}$ before, but we have to change notation here due to the primes in the target of $\Phi)$.

First, we claim that we can precompose with an automorphism of the source such that $\Phi^{*}\left(t^{\prime}\right)=t$. Write $\Phi^{*}\left(t^{\prime}\right)=t f$ for some degree-zero function $f$ which is nonvanishing at the ideal $\tilde{J}$ of the punctured line $L:=\mathbf{C}^{\times} \times\{0\} \times\{0\}$. Therefore $f$ is invertible. Up to composing with an isomorphism $t \mapsto \lambda t, u \mapsto \lambda^{-1} u$ for some $\lambda \in \mathbf{C}^{\times}$(which is the identity on $z_{i}$ and on $S$ ) we can assume that $f \in 1+\tilde{J}$. Set $f_{1}:=f$. We prove by induction that there is a sequence $\left(f_{1}, f_{2}, \ldots\right)$ of elements $f_{q} \in 1+(\tilde{J})^{q}$ and a sequence of 
$\mathbf{C}^{\times}$-Poisson automorphisms $\left(\Psi_{1}, \Psi_{2}, \ldots\right)$ such that $\Psi_{q}^{*}$ is the identity modulo $(\tilde{J})^{q}$ and takes $t f_{q}$ to $t f_{q+1}$. Then the composition $\cdots \Psi_{2}^{*} \Psi_{1}^{*} \Phi^{*}$ converges to a graded continuous Poisson algebra isomorphism taking $t^{\prime}$ to $t$, proving the claim.

Since continuous Poisson algebra automorphisms which are the identity modulo $(\tilde{J})^{q}$ are all exponentials of Poisson vector fields which are zero modulo $(\tilde{J})^{q}$, the statement is equivalent to finding Poisson vector fields $\xi_{q}$ which are zero modulo $(\tilde{J})^{q}$ such that

$$
\xi\left(t f_{q}\right)+t f_{q} \equiv t \quad\left(\bmod (\tilde{J})^{q+1}\right) .
$$

In fact, we have Hamiltonian vector fields with this property, by the identity

$$
\left\{t^{k} \int\left(f_{q}-1\right) d u, t f_{q}\right\} \equiv t\left(1-f_{q}\right) f_{q} \equiv t-t f_{q} \quad\left(\bmod (\tilde{J})^{q+1}\right),
$$

where $\int\left(f_{q}-1\right) d u$ is the unique antiderivative of $\left(f_{q}-1\right)$ with respect to $u$ which is a multiple of $u$ (note that $\int\left(f_{q}-1\right) d u \in(\tilde{J})^{q+1}$.)

Next, we may suppose that the Poisson bivectors of $S$ and $S^{\prime}$ vanish at the origin; otherwise we could decompose $S$ and $S^{\prime}$ as products of $\Delta^{2 r}$ by some formal Poisson schemes with Poisson bivectors vanishing at the origin, where $2 r$ is the rank of the Poisson bivectors of $S$ and $S^{\prime}$ at the origin (which must be equal since the ranks of the Poisson bivectors on the products $\mathbf{C}^{\times} \times \Delta^{2 m-1} \times S$ and $\mathbf{C}^{\times} \times \Delta^{2 m-1} \times S$ are $\left.2 r+2 m\right)$. In this case $\Phi$ must preserve the tangent spaces to $\mathbf{C}^{\times} \times \Delta^{2 m-1}$ since these tangent spaces are the spans of the Hamiltonian vector fields. Up to change of coordinates, we can assume that $\Phi$ acts as the identity on this tangent space (by the proof of Theorem 19).

A standard argument used to show uniqueness of the slices in the usual formal DarbouxWeinstein decomposition shows that we can apply Hamiltonian isomorphisms so that the coordinates $z_{1}^{\prime}, \ldots, z_{2 m-2}^{\prime}$ map to $z_{1}, \ldots, z_{2 m-2}$. Namely, suppose that $\Phi^{*}\left(z_{k}^{\prime}\right)-z_{k} \in(\tilde{J})^{q+1}$ for $k<2 i-1$ and $\Phi^{*}\left(z_{k}^{\prime}\right)-z_{k} \in(\tilde{J})^{q}$ for $k \geq 2 i-1$. Then write $f:=\Phi^{*}\left(z_{2 i-1}^{\prime}\right)-z_{2 i-1} \in(\tilde{J})^{q}$. For $k<2 i-1$, we have

$$
\left\{f, z_{k}\right\}=\left\{\Phi^{*}\left(z_{2 i-1}\right), z_{k}\right\}=\left\{\Phi^{*}\left(z_{2 i-1}\right), \Phi^{*}\left(z_{k}\right)-z_{k}\right\} \in(\tilde{J})^{q} .
$$

Then, we can apply the Hamiltonian isomorphism $\exp \left(\xi_{\int f d z_{2 i}}\right)$, which is the identity modulo $(\tilde{J})^{q}$, leaves $\Phi^{*}\left(z_{k}^{\prime}\right)$ unchanged modulo $(\tilde{J})^{q+1}$ for $k<2 i-1$, and takes $\Phi^{*}\left(z_{2 i-1}\right)$ to an element equivalent to $z_{2 i-1}$ modulo $(\tilde{J})^{q+1}$. Similarly, if then $\Phi^{*}\left(z_{2 i}\right)=z_{2 i}+g$ for $g \in(\tilde{J})^{q}$, we can then apply $\exp \left(\xi_{-\int g d z_{2 i-1}}\right)$. After this, we will have that $\Phi^{*}\left(z_{k}^{\prime}\right)-z_{k} \in(\tilde{J})^{q+1}$ for $k<2 i+1$ and $\Phi^{*}\left(z_{k}^{\prime}\right)-z_{k} \in(\tilde{J})^{q}$ for $k \geq 2 i+1$, completing the induction. Moreover, since $\Phi\left(t^{\prime}\right)=t$, we had $\left\{\Phi\left(z_{i}^{\prime}\right), t\right\}=0$, which shows that the above procedure will preserve $t$.

We may therefore assume that $\Phi^{*}\left(t^{\prime}\right)=t$ and $\Phi^{*}\left(z_{i}^{\prime}\right)=z_{i}$ for all $i$. Thus $\Phi^{*}\left(u^{\prime}\right)=u+f$ for some $f$ in the augmentation ideal of $\mathcal{O}_{S}$. It follows that $\mathcal{O}_{S^{\prime}}$, which is the weight-zero part of the centralizer of $t^{\prime}, z_{1}^{\prime}, \ldots, z_{2 m-2}^{\prime}$, must map to $\mathcal{O}_{S}$. Thus $\Phi$ produces a Poisson isomorphism $S \rightarrow S^{\prime}$, as desired.

Finally if we compose with a continuous graded Poisson algebra automorphism fixing $t, z_{1}, \ldots, z_{2 m}$ and sending $u$ to $u+f$ (for $f$ in the augmentation ideal), we see that $f$ must 
actually be in the augmentation ideal of $\mathcal{O}_{S}$. That is, this changes $\xi$ appearing in (36) to $\xi+t^{-k} \xi_{f}$, i.e., $\xi+\xi_{f}^{\prime}$ where $\xi_{f}^{\prime}$ is the Hamiltonian vector field on $\left(S, \pi_{S}\right)$ associated to $f$. Conversely given any $f$, we can apply the automorphism which is the identity on $t, z_{i}$, and $\mathcal{O}_{S}$, sending $u$ to $u+f$, which is Poisson from the bivector in (36) to the one replacing $\xi$ with $\xi+\xi_{f}^{\prime}$.

\subsection{Proof of Theorem 43}

For most of the proof, we follow (and generalize) the proof of Proposition [PS16, Proposition $5.5]$.

We first show that the hypothesis of (i) implies (ii). In the more general situation where $X$ is a symplectic singularity, this is actually a consequence of [Xu14], as pointed out in Nam13b: there it is shown that, for a small enough neighborhood $U$ of $y$, the algebraic fundamental group $\hat{\pi}_{1}\left(U^{\circ}\right)$ of the smooth locus $U^{\circ}$ of $U$ is finite. This in particular implies that the fundamental group $\pi_{1}\left(U^{\circ}\right)$ cannot have $\mathbf{Z}$ as a quotient, so that $H_{1}\left(U^{\circ}\right)$ is torsion. Thus, $H_{1}\left(U^{\circ}, \mathbf{C}\right)=0$, and hence $H^{1}\left(U^{\circ}, \mathbf{C}\right)=0$, as desired.

However, we give a more direct proof (using only [Kal06, Theorem 2.12]) in the case that $X$ has a (projective) symplectic resolution. Note that, by [Kal06, Theorem 2.12], if $\rho: \tilde{X} \rightarrow X$ is a symplectic resolution, then $H^{1}\left(\rho^{-1}(y), \mathbf{C}\right)=0$. Take some tubular analytic neighborhood of $\rho^{-1}(y)$ which contracts to $\rho^{-1}(y)$. Since $\rho^{-1}(y)$ is compact, there is some open ball $U$ around $y$ such that $\rho^{-1}(U)$ lands in the tubular neighborhood and therefore contracts to $\rho^{-1}(y)$. Thus $H^{1}\left(\rho^{-1}(U), \mathbf{C}\right)=0$.

Let $U^{\circ}$ be the smooth locus of $U$, and write $U \backslash U^{\circ}=\bigcup_{i} U_{i}$ for some closed subsets $U_{i} \subseteq U$. Since $\rho$ is an isomorphism over $U^{\circ}$, it suffices to show that $H^{1}\left(\rho^{-1}\left(U^{\circ}\right), \mathbf{C}\right)$. By the semismallness property [Kal06, Lemma 2.11] of $\rho$, the complex codimension of $\rho^{-1}\left(U_{i}\right)$ is at least half the codimension of $U_{i}$. Thus, the real codimension of $\rho^{-1}\left(U_{i}\right)$ is at least equal to the complex codimension of $U_{i}$. Since the fundamental group of a smooth manifold is unchanged by removing a locus of real codimension greater than two (as all homotopies can be pushed away from the removed locus), we conclude that the fundamental group of $\rho^{-1}\left(U^{\circ}\right)$ equals that of $\rho^{-1}(U) \backslash \sqcup_{\operatorname{dim}_{\mathbf{C}} U_{i}=\operatorname{dim}_{\mathbf{C}} U-2} \rho^{-1}\left(U_{i}\right)$. Moreover, since we can homotope any path in $U$ away from a locus of real codimension two, the map $\pi_{1}\left(\rho^{-1}\left(U^{\circ}\right)\right) \rightarrow \pi_{1}\left(\rho^{-1}(U)\right)$ is surjective. For each $U_{i}$ of complex codimension two, the singularity at each $y \in U_{i}$ is of Kleinian type. The fundamental group of the smooth locus of a Kleinian singularity is finite (it is the corresponding group $\Gamma<\operatorname{SL}(2, \mathbf{C}))$. Therefore, the kernel of $\pi_{1}\left(\rho^{-1}\left(U^{\circ}\right)\right) \rightarrow \pi_{1}\left(\rho^{-1}(U)\right)$ is generated by torsion elements. Thus, $H_{1}\left(\rho^{-1}(U), \mathbf{Z}\right)$ is a quotient of $H_{1}\left(\rho^{-1}\left(U^{\circ}\right), \mathbf{Z}\right)$ by torsion, and $H_{1}\left(\rho^{-1}\left(U^{\circ}\right), \mathbf{C}\right) \rightarrow H_{1}\left(\rho^{-1}(U), \mathbf{C}\right)$ is an isomorphism. Since $H_{1}\left(\rho^{-1}(U), \mathbf{C}\right)=0$, also $H_{1}\left(\rho^{-1}\left(U^{\circ}\right), \mathbf{C}\right)=0$, and hence $H^{1}\left(\rho^{-1}\left(U^{\circ}\right), \mathbf{C}\right)=0$. Thus $H^{1}\left(U^{\circ}, \mathbf{C}\right)=0$, as desired.

Next, we explain why part (ii) implies (iii). For this we can assume $S$ comes from an ordinary Darboux-Weinstein decomposition; otherwise completing at $y$, we still get a product of formal schemes $\hat{X}_{y} \cong \hat{Y}_{y} \times S$ (it is just not necessarily Poisson), and this is all we will use. Assume (ii). Let $\rho: V \rightarrow U$ be an arbitrary resolution of singularities (not necessarily symplectic). Assume $U$ is small enough that $V$ contracts to $\rho^{-1}(y)$. Let $Z \subseteq U$ be the singular locus, so $U^{\circ}=U \backslash Z$. Let $\mathbf{H}_{D R}^{\bullet}$ denote the de Rham hypercohomology. 
By Hartshorne's theorem [Har72, $\operatorname{Har75}, \mathbf{H}_{D R}^{\bullet}\left(\hat{V}_{\rho^{-1}(y)}\right) \cong H^{\bullet}\left(\rho^{-1}(U)\right) \cong H^{\bullet}\left(\rho^{-1}(y)\right)$, the topological cohomology. Applying the Mayer-Vietoris sequence (cf. [ES16, (4.40)-(4.44)]), we find that $\mathbf{H}_{D R}^{\bullet}\left(\hat{V}_{\rho^{-1}(y)} \backslash \rho^{-1}(Z)\right) \cong H^{\bullet}\left(\rho^{-1}(U) \backslash \rho^{-1}(Z)\right) \cong H^{\bullet}\left(U^{\circ}\right)$. On the other hand, $\hat{V}_{\rho^{-1}(y)} \backslash \rho^{-1}(Z)=\hat{U}_{y} \backslash Z$, since $\rho$ is an isomorphism over $U^{\circ}=U \backslash Z$. Thus we conclude that $\mathbf{H}_{D R}^{\bullet}\left(\hat{U}_{y} \backslash Z\right) \cong H^{\bullet}\left(U^{\circ}\right)$. Now, $\tilde{\Omega}_{\hat{U}_{y}}$ is the global sections of the algebraic de Rham complex on $\hat{U}_{y} \backslash Z$ (since $U \backslash Z$ is the smooth locus of $U, U$ is normal, and $Z$ has codimension at least two). Therefore, by the spectral sequence computing hypercohomology, $H^{1}\left(\tilde{\Omega}_{\hat{U}_{y}}\right)$ is a summand of $\mathbf{H}_{D R}^{1}\left(\hat{U}_{y} \backslash Z\right)=H^{1}\left(U^{\circ}\right)$ (alternatively, we can see that the canonical map $H^{1}\left(\tilde{\Omega}_{\hat{U}_{y}}\right) \rightarrow H^{1}\left(U^{\circ}\right)$ is injective since any algebraic one-form which is the differential of a smooth function is actually the differential of an algebraic function, and the same applies after taking formal completions). By the hypothesis, the latter is zero, establishing $H^{1}\left(\tilde{\Omega}_{\hat{U}_{y}}^{\bullet}\right)=0$. Since $\hat{U}_{y} \cong \Delta^{\operatorname{dim} Y} \times S$, we also get $H^{1}\left(\tilde{\Omega}_{S}^{\bullet}\right)=0$, as desired.

Finally, we explain why part (iii) implies (iv). Suppose $H^{1}\left(\tilde{\Omega}_{S}^{\bullet}\right)=0$ and $X$ (hence $S$ ) is generically symplectic. If $\xi$ is a Poisson vector field on $S$, i.e., $[\xi, \pi]=0$ for $\pi$ the Poisson structure, then for $\omega=\pi^{-1}$ the generic symplectic structure, $i_{\xi} \omega$ is a closed one-form on the smooth locus of $S$, and it is exact if and only if it is Hamiltonian.

\subsection{Quantization}

Parallel to Theorem 26, Theorem 34 has the following consequence. Let $\mathcal{D}_{\hbar, k}\left(\mathbf{C}^{\times} \times \Delta^{n-1}\right)$ denote the completion of (27) with respect to the ideal $\left(\hbar, u, z_{1}^{\prime}, \ldots, z_{2 n-2}^{\prime}\right)$, i.e., the quantization of $\mathbf{C}^{\times} \times \Delta^{2 n-1}$ discussed in $\$ 2.2$.

Theorem 48. Let $X, Y, y$, and $k$ be as in Theorem 34, Then, every $\mathbf{C}^{\times}$-compatible quantization $A_{\hbar}$ of $\hat{X}_{\mathbf{C}^{\times} \cdot y}$ has subalgebras $\mathcal{D}_{\hbar, k}\left(\mathbf{C}^{\times} \times \Delta^{(\operatorname{dim} Y) / 2-1}\right)$ and $A_{\hbar}^{\prime}$ quantizing $\hat{Y}_{\mathbf{C}^{\times} \cdot y}$ and $\mathbf{C}^{\times} \times S$, respectively, so that $A_{\hbar}^{\prime}$ is the simultaneous centralizer of $t, z_{1}, \ldots, z_{\operatorname{dim} Y-2}$. We have

$$
A_{\hbar} \cong \mathcal{D}_{\hbar, k}\left(\mathbf{C}^{\times} \times \Delta^{(\operatorname{dim} Y) / 2-1}\right) \hat{\otimes}_{\mathbf{C}\left[t, t^{-1}\right][\hbar \rrbracket} A_{\hbar}^{\prime}
$$

as graded topological $\mathbf{C} \llbracket \hbar \rrbracket$-modules.

In the case that all Poisson vector fields on $S$ are Hamiltonian (or any of the conditions of Theorem 43 are satisfied), then we can choose the coordinate $u$ so that (49) is a graded algebra isomorphism. If, further, the conditions of Corollary 44 are satisfied, i.e., $S$ also admits a $\mathbf{C}^{\times}$action giving its Poisson structure degree $-k$, and this action quantizes to a compatible action on $A_{\hbar}^{\prime}$ (now acting trivially on $\mathbf{C}\left[t, t^{-1}\right] \subseteq A_{\hbar}^{\prime}$ ), then we can replace the tensor product above with one over $\mathbf{C} \llbracket \hbar \rrbracket$ : for $A_{\hbar}^{\prime \prime} \subseteq A_{\hbar}^{\prime}$ the subspace where the two $\mathbf{C}^{\times}$ actions have the same weight,

$$
A_{\hbar} \cong \mathcal{D}_{\hbar, k}\left(\mathbf{C}^{\times} \times \Delta^{(\operatorname{dim} Y) / 2-1}\right) \hat{\otimes}_{\mathbf{C} \llbracket \hbar \rrbracket} A_{\hbar}^{\prime \prime},
$$

as graded algebras.

Motivated by the additional hypothesis in the second paragraph of Theorem 48, we can ask the following quantum analogue of Question 3 , 
Question 51. Suppose $X$ is conical and admits a $\mathbf{C}^{\times}$-equivariant symplectic resolution with homogeneous symplectic form of degree $k$ (or is a symplectic singularity and has a symplectic structure of degree $k$ on the smooth locus). Let $x \in X$ have trivial stabilizer under $\mathbf{C}^{\times}$and suppose that Question 3 has a positive answer, i.e., the $S$ in (2) admits a contracting $\mathbf{C}^{\times}$ action for which the Poisson structure is homogeneous, and suppose it has degree $k$. Then, does every $\mathbf{C}^{\times}$-compatible quantization of $X$ contain a subalgebra which quantizes $S$ and admits a compatible lift of the $\mathbf{C}^{\times}$action on $S$ ?

We see in the next sections that this question has a positive answer, at least, for certain quantizations of linear quotients and hypertoric varieties. Note, as pointed out after the statement of Question 3, that the degree $k$ must be positive in Question 51 .

Remark 52. As in Remark 4, one can also weaken the hypotheses of the question and require only that $X$ be normal and conical and have a symplectic form of positive degree $k$ on its smooth locus (and not require that $X$ be a symplectic singularity).

Proof of Theorem 48. By Theorem 34, it is enough to replace $\hat{X}_{\mathbf{C}^{\times} \cdot y}$ with $\left(\mathbf{C}^{\times} \times \Delta^{2 n-1}\right) \times S$, with Poisson bivector (36). We moreover view the quantization as an associative star product on $\mathcal{O}\left(\mathbf{C}^{\times} \times \Delta^{2 n-1} \times S\right) \llbracket \hbar \rrbracket$. In this paragraph, we apply arguments similar to those in $₫ 2.1$ and in the proof of Theorem 26 in order to find a gauge transformation taking $t, u, z_{1}, \ldots, z_{2 n-2}$ to coordinates satisfying the desired commutation relations; this will actually be surprisingly simple since the commutators are already correct modulo $\hbar^{2}$ (and this will produce a slightly different, more explicit, proof of Theorem [26). We continue to use the $z_{i}^{\prime}$ defined as before, $z_{i}^{\prime}=z_{i}$ for $i$ even and $z_{i}^{\prime}=t^{k} z_{i}$ for $i$ odd). Let $[a, b]_{\star}:=a \star b-b \star a$. To begin, we know that commutators of these coordinates are correct modulo $\hbar^{2}$. Suppose that they are correct modulo $\hbar^{m}$ for some $m \geq 2$. Then, we can apply coordinate changes $u \mapsto$ $u-\hbar^{-1} t^{k-1} \int\left([t, u]_{\star}-\hbar t^{1-k}\right) d u, z_{i} \mapsto z_{i}-\hbar^{-1} t^{k-1} \int\left[t, z_{i}\right]_{\star} d u$ for all $i$ so that the commutators with $t$ are now correct modulo $\hbar^{m+1}$. Similarly, for $i$ even and between 2 and $2 n-2$, we can apply coordinate changes $u \mapsto u-\hbar^{-1} t^{k} \int\left[u, z_{i}^{\prime}\right]_{\star} d z_{i-1}$ and for $i$ odd, $u \mapsto u+\hbar^{-1} \int\left[u, z_{i}^{\prime}\right]_{\star} d z_{i+1}$, so that the commutators with $u$ are correct modulo $\hbar^{m+1}$. Finally, with $j>i$, we can apply coordinate changes $z_{j} \mapsto z_{j}-\hbar^{-1} t^{k} \int\left[z_{j}, z_{i}\right]_{\star} d z_{i-1}$ for $i$ even, $z_{j} \mapsto z_{j}+\hbar^{-1} t^{k} \int\left[z_{j}, z_{i}\right]_{\star} d z_{i+1}$ when $i$ is odd and $j \neq i+1$, and finally $z_{2 i} \mapsto z_{2 i}+\hbar^{-1} t^{k} \int\left(\left[z_{2 i-1}, z_{2 i}\right]_{\star} d z_{2 i}\right.$ for all $i$, after which all commutators among the $t, u, z_{1}, \ldots, z_{2 n-2}$ will be correct modulo $\hbar^{m+1}$.

We now assume that the $t, u, z_{1}, \ldots, z_{2 n-2}$ have the correct commutation relations. They therefore generate the desired subalgebra $\mathcal{D}_{\hbar, k}\left(\mathbf{C}^{\times} \times \Delta^{(\operatorname{dim} Y) / 2-1}\right)$ quantizing $\hat{Y}_{\mathbf{C}^{\times} \cdot y}$. We define $A_{\hbar}^{\prime}$ as the subalgebra of elements commuting with $t, z_{1}, \ldots, z_{2 n-2}$. We need to show that (49) holds. To do this, we will show that there is a continuous graded $\mathbf{C} \llbracket \hbar \rrbracket$-linear map $\Phi: \mathcal{O}\left(S \times \mathbf{C}^{\times}\right) \llbracket \hbar \rrbracket \rightarrow \mathcal{O}\left(\mathbf{C}^{\times} \times \Delta^{2 n-1} \times S\right) \llbracket \hbar \rrbracket$, which is the inclusion modulo $\hbar$, such that the image of $\Phi$ is $A_{\hbar}^{\prime}$. Taking the product of this with the inclusion we obtain a continuous $\mathbf{C} \llbracket \hbar \rrbracket$ linear map $\mathcal{O}\left(\mathbf{C}^{\times} \times \Delta^{2 n-1} \times S\right) \llbracket \hbar \rrbracket \rightarrow \mathcal{O}\left(\mathbf{C}^{\times} \times \Delta^{2 n-1} \times S\right) \llbracket \hbar \rrbracket$ which is an isomorphism modulo $\hbar$, hence an isomorphism of graded topological $\mathbf{C} \llbracket \hbar \rrbracket$-modules. It sends $\mathcal{O}\left(\mathbf{C}^{\times} \times \Delta^{2 n-1}\right) \llbracket \hbar \rrbracket$ to $\mathcal{D}_{\hbar, k}\left(\mathbf{C}^{\times} \times \Delta^{(\operatorname{dim} Y) / 2-1}\right)$ and $\mathcal{O}\left(S \times \mathbf{C}^{\times}\right) \llbracket \hbar \rrbracket$ to $A_{\hbar}^{\prime}$. Thus, we will conclude (49).

We construct $\Phi$ order by order. For every $m \geq 1$, let $\left(A_{\hbar}^{\prime}\right)_{m} \subseteq \mathcal{O}\left(\mathbf{C}^{\times} \times \Delta^{2 n-1} \times\right.$ $S)[\hbar] /\left(\hbar^{m+1}\right)$ be the subalgebra of elements annihilated by the operators $\hbar^{-1} \operatorname{ad}(t)$ and 
$\hbar^{-1}$ ad $\left(z_{i}\right)$ for all $i$. Assume that, for some $m \geq 1$, there exists $\Phi_{m}: \mathcal{O}\left(S \times \mathbf{C}^{\times}\right)[\hbar] /\left(\hbar^{m+1}\right) \rightarrow$ $\mathcal{O}\left(\mathbf{C}^{\times} \times \Delta^{2 n-1} \times S\right)[\hbar] /\left(\hbar^{m+1}\right)$ with image $\left(A_{\hbar}^{\prime}\right)_{m}$, which is the identity modulo $\hbar$. We must extend this to a map $\Phi_{m+1}$ with image $\left(A_{\hbar}^{\prime}\right)_{m+1}$. Note first that the intersection $\left(A_{\hbar}^{\prime}\right)_{m+1} \cap \hbar^{m+1} \mathcal{O}\left(\mathbf{C}^{\times} \times \Delta^{2 n-1} \times S\right)[\hbar] /\left(\hbar^{m+2}\right)$ is $\hbar^{m+1} \cdot \mathcal{O}\left(\mathbf{C}^{\times} \times S\right)$, since the condition for a multiple of $\hbar^{m+1}$ to be annihilated by $\hbar^{-1}$ ad $(f)$ modulo $\hbar^{m+2}$ is just the condition that the element Poisson commute with $f$, for all $f \in \mathcal{O}\left(\mathbf{C}^{\times} \times \Delta^{2 n-1} \times S\right)$, and we apply this to $f \in\left\{t, z_{1}, \ldots, z_{2 n-2}\right\}$. Therefore, the existence of $\Phi_{m+1}$ is equivalent to the statement that every element $a \in\left(A_{\hbar}^{\prime}\right)_{m}$ has a lift $\tilde{a} \in\left(A_{\hbar}^{\prime}\right)_{m+1}$ to an element of $\left(A_{\hbar}^{\prime}\right)_{m+1}$. This in turn is equivalent to the existence of a solution $a_{m+1} \in \mathbf{C}\left(\mathbf{C}^{\times} \times \Delta^{2 n-1} \times S\right)$ to the equations $\hbar^{-1}\left[t, \hbar^{m+1} a_{m+1}\right]_{\star} \equiv \hbar^{-1}[t, a]_{\star}\left(\bmod \hbar^{m+2}\right)$ and $\hbar^{-1}\left[z_{i}^{\prime}, \hbar^{m+1} a_{m+1}\right]_{\star} \equiv \hbar^{-1}\left[z_{i}^{\prime}, a\right]_{\star}\left(\bmod \hbar^{m+2}\right)$ for all $i$.

Let $\operatorname{ad}_{\star}(f)$ be the operator $\operatorname{ad}_{\star}(f)(g)=[f, g]_{\star}$. Define the operators $\nabla_{u}:=\hbar^{-1} t^{k-1} \operatorname{ad}_{\star}(t)$, $\nabla_{z_{i}}:=-\hbar^{-1} t^{k} \operatorname{ad}_{\star}\left(z_{i+1}\right)$ for $i$ odd, and $\nabla_{z_{i}}:=\hbar^{-1} \operatorname{ad}_{\star}\left(z_{i-1}^{\prime}\right)$ for $i$ even. These operators satisfy the identities $\nabla_{u}(u \star f)=u \star \nabla_{u}(f)+f$ and $\nabla_{z_{i}}\left(z_{i} \star f\right)=z_{i} \star \nabla_{z_{i}} f+f$. Moreover, the operators commute, by the Jacobi identity for commutators. The above equations are equivalent to

$$
\nabla_{u}\left(a_{m+1}\right) \equiv \hbar^{-m-1} \nabla_{u}(a) \quad(\bmod \hbar), \nabla_{z_{i}}\left(a_{m+1}\right) \equiv \hbar^{-m-1} \nabla_{z_{i}}(a) \quad(\bmod \hbar) \forall i .
$$

Modulo $\hbar$, the operators $\nabla_{u}, \nabla_{z_{i}}$ define a flat connection on $\mathbf{C}\left(\mathbf{C}^{\times} \times \times \Delta^{2 n-1} \times S\right)$ over $\mathbf{C} \llbracket u, z_{1}, \ldots, z_{2 n-1} \rrbracket=\mathbf{C}\left(\Delta^{2 n-1}\right)$. Writing the above equations as $\nabla_{u}\left(a_{m+1}\right) \equiv F_{u}(\bmod \hbar)$ and $\nabla_{z_{i}}\left(a_{m+1}\right)=F_{z_{i}}(\bmod \hbar)$, it follows from the previous identities that the integrability conditions $\nabla_{z_{i}} F_{u}=\nabla_{u} F_{z_{i}}$ and $\nabla_{z_{i}} F_{z_{j}}=\nabla_{z_{j}} F_{z_{i}}$ hold. Therefore there exist solutions to the above equations. This completes the proof of (49).

In the case that all Poisson vector fields on $S$ are Hamiltonian, we can inductively replace $u$ by a new coordinate function (which remains unchanged modulo $\hbar$ ), so that $u$ commutes with $A_{\hbar}^{\prime}$ and continues to satisfy the needed commutation relations in $\mathcal{D}_{\hbar, k}\left(\mathbf{C}^{\times} \times \Delta^{(\operatorname{dim} Y) / 2-1}\right)$. We begin, by Corollary 44 , knowing $\left[u, A_{\hbar}^{\prime}\right] \subseteq \hbar^{2} A_{\hbar}$. Assume $\left[u, A_{\hbar}^{\prime}\right] \subseteq \hbar^{N} A_{\hbar}$ for some $N \geq 2$. Then we can consider the vector field $\hbar^{-N} t^{k N}$ ad $(u)$ on $\mathcal{O}_{S}$ valued in $A_{\hbar}$. In fact, it must be valued in the centralizer of $t, u, z_{1}^{\prime}, \ldots, z_{2 n-2}^{\prime}$, by the Jacobi identity, so it is valued in $A_{\hbar}^{\prime}$. Then, it is Hamiltonian, so we can subtract an element of $\hbar^{N-1} A_{\hbar}^{\prime}$ from $u$ so that $\left[u, A_{\hbar}^{\prime}\right] \subseteq \hbar^{N+1} A_{\hbar}$.

Finally, if the assumptions of Corollary 44 are satisfied and $A_{\hbar}^{\prime}$ has a $\mathbf{C}^{\times}$-compatible action for the new grading (giving $t$ now degree zero), then it remains to note that $A_{\hbar}^{\prime \prime}$ as defined in the theorem is a graded subalgebra of $A_{\hbar}^{\prime}$, and that $A_{\hbar}^{\prime}=A_{\hbar}^{\prime \prime}\left[t, t^{-1}\right]$.

\section{$4 \quad$ Finite linear quotients}

In this section we give more explicit and stronger versions of the main theorem when $X$ is a finite quotient of a symplectic vector space. In particular, we explain how one can replace formal localization by an explicit étale (or Zariski) localization.

Let $V$ be a symplectic vector space and $\Gamma<\mathrm{Sp}(V)$ a finite subgroup. We consider the quotient $X:=V / \Gamma$. The symplectic structure on $V$ makes $X$ a Poisson variety. Equip $V$ 
with the dilation action of $\mathbf{C}^{\times}$, which makes its symplectic form have weight 2 , and hence the Poisson structures on $V$ and $X$ have weight -2 .

Recall that a subgroup $\Gamma_{0}<\Gamma$ is called parabolic if there exists $v \in V$ such that $\operatorname{Stab}_{\Gamma}(v)=\Gamma_{0}$. Let $V^{\Gamma_{0}}$ be the fixed point set of $\Gamma_{0}, N\left(\Gamma_{0}\right)<\Gamma$ be the normalizer, and $N\left(\Gamma_{0}\right)^{0}:=N\left(\Gamma_{0}\right) / \Gamma_{0}$, the residual action on $V^{\Gamma_{0}}$. Then one has the symplectic leaf $X_{\Gamma_{0}}:=\left(V^{\Gamma_{0}}\right)^{\circ} / N\left(\Gamma_{0}\right)^{0}$, where $\left(V^{\Gamma_{0}}\right)^{\circ}:=\left\{w \in V \mid \operatorname{Stab}_{\Gamma}(w)=\Gamma_{0}\right\}$. Its closure is $\bar{X}_{\Gamma_{0}}=V^{\Gamma_{0}} / N\left(\Gamma_{0}\right)^{0}$. Moreover, all symplectic leaves $X_{\Gamma_{0}}$ are obtained in this way, and this establishes a bijection between conjugacy classes of parabolic subgroups of $\Gamma$ and symplectic leaves (or symplectic leaf closures) of $X$.

Let $\Gamma_{0}<\Gamma$ be a parabolic subgroup. Fix $v \in V^{\Gamma_{0}}$ with stabilizer equal to $\Gamma_{0}$, and let $\bar{v} \in X_{\Gamma_{0}}$ be its image in $X$. We assume (as in Theorem 1) that $\mathbf{C}^{\times}$acts freely on $\bar{v}$, i.e., that $v$ is not an eigenvector of any nontrivial element of $N\left(\Gamma_{0}\right)^{0} \cdot 5$

Proposition 53. There is a canonical $\mathbf{C}^{\times}$-equivariant Poisson isomorphism

$$
\hat{X}_{\mathbf{C}^{\times} \cdot \bar{v}} \cong\left(\widehat{X_{\Gamma_{0}}}\right)_{\mathbf{C}^{\times} \cdot \bar{v}} \times \widehat{\left(V^{\Gamma_{0}}\right)^{\perp}} / \Gamma_{0} .
$$

This implies that Question 3 has an affirmative answer in this case.

The proposition follows easily from the following étale local statement. Let $V^{\circ}:=\{w \in$ $\left.V \mid \operatorname{Stab}_{\Gamma}(w)<\Gamma_{0}\right\}$ and denote by $X^{\circ}=V^{\circ} / \Gamma$ its image. Then we have an étale cover $V^{\circ} / \Gamma_{0} \rightarrow X^{\circ}$. Note that $X_{\Gamma_{0}}$ is closed in $X^{\circ}$. We have an étale covering $\left(V^{\Gamma_{0}}\right)^{\circ} \rightarrow X_{\Gamma_{0}}$. Clearly, passing to the étale covers,

$$
V^{\circ} / \Gamma_{0} \cong\left(V^{\Gamma_{0}}\right)^{\circ} \times\left(V^{\Gamma_{0}}\right)^{\perp} / \Gamma_{0}
$$

We note that this holds for all $v \in\left(V^{\Gamma_{0}}\right)^{\circ}$. Then, the proposition follows from this together with the fact that, when $\mathbf{C}^{\times}$acts freely on $\bar{v}$, then the natural map $\widehat{V^{\Gamma_{0}}} \mathbf{C}^{\times} \cdot v+\left(\widehat{X_{\Gamma_{0}}}\right)_{\mathbf{C}^{\times} \cdot v}$ of completions is an isomorphism (since $\mathbf{C}^{\times} \cdot v$ does not intersect its image under any nontrivial element of $\left.N\left(\Gamma_{0}\right)^{0}\right)$.

Remark 55. Putting the above together with the results of Section 3, we can relate the Darboux-Weinstein decompositions for an arbitrary $\mathbf{C}^{\times}$-Poisson variety $X$ with a quotient $X / \Gamma$ for $\Gamma$ a finite subgroup. Namely, suppose we are given $y$ in a symplectic leaf $Y \subseteq X$ which we assume to be $\mathbf{C}^{\times}$-stable and a $\mathbf{C}^{\times}$-equivariant Poisson decomposition $\hat{X}_{\mathbf{C}^{\times} \cdot y} \cong$ $\hat{Y}_{\mathbf{C}^{\times} \cdot y} \times S$. As before, let $\Gamma_{0}:=\operatorname{Stab}_{\Gamma}(y), N\left(\Gamma_{0}\right)<\Gamma$ be the normalizer, and $N\left(\Gamma_{0}\right)^{0}:=$ $N\left(\Gamma_{0}\right) / \Gamma_{0}$. Let $z:=\Gamma \cdot y \in X / \Gamma$. Then the symplectic leaf of $z$ in $X / \Gamma$ is $Z:=Y_{\Gamma_{0}} / N\left(\Gamma_{0}\right)^{0}$ for $Y_{\Gamma_{0}}:=\left\{y^{\prime} \in Y \mid \operatorname{Stab}_{\Gamma}\left(y^{\prime}\right)=\Gamma_{0}\right\}$. Assume that $\mathbf{C}^{\times}$acts freely on $z$. Then, we then get a decomposition $\widehat{X / \Gamma_{\mathbf{C}^{\times} \cdot z}} \widehat{Z}_{\mathbf{C}^{\times} \cdot z} \times\left(\left(T_{y} Y_{\Gamma_{0}}\right)^{\perp} \times S\right) / \Gamma_{0}$, for $\left(T_{y} Y_{\Gamma_{0}}\right)^{\perp} \subseteq T_{y} Y$ the perpendicular to $T_{y} Y_{\Gamma_{0}}$.

\footnotetext{
${ }^{5}$ Note that such a $v \in V^{\Gamma_{0}}$ always exists unless - Id $\in N\left(\Gamma_{0}\right)^{0}$, since no multiples of the identity other than \pm Id preserve the symplectic form on $V^{\Gamma_{0}}$. In other words, the $\ell$ appearing in Theorem 34 must be either two or one, and it is two if and only if $-\mathrm{Id} \in N\left(\Gamma_{0}\right)^{0}$.
} 
Remark 56. In the case above where $V=T^{*} U$ for $U$ a complex vector space and $\Gamma<$ $\mathrm{GL}(U)<\operatorname{Sp}(V)$, we can strengthen the proposition by completing only in the $U$ direction. For $u \in U$ with stabilizer $\Gamma_{0}$, and $\bar{u} \in U / \Gamma \subseteq X$ its image, this yields

$$
\begin{aligned}
& X \times_{U / \Gamma} \widehat{U / \Gamma}_{\mathbf{C}^{\times} \cdot \bar{u}} \cong \\
& \quad\left(X_{\Gamma_{0}} \times_{U^{\Gamma_{0}} / N\left(\Gamma_{0}\right)^{0}} U^{\Gamma_{0}} \widehat{\left.\widehat{N N\left(\Gamma_{0}\right.}\right)^{0}}{ }_{\mathbf{C}^{\times} \cdot \bar{u}}\right) \times\left(T^{*}\left(U^{\Gamma_{0}}\right)^{\perp} / \Gamma_{0} \times_{\left(U^{\left.\Gamma_{0}\right)^{\perp}} / \Gamma_{0}\right.}\left(\widehat{\left.U^{\Gamma_{0}}\right)^{\perp}} / \Gamma_{0}\right) .\right.
\end{aligned}
$$

Also, in the case that $N\left(\Gamma_{0}\right)^{0}=\{1\}$, then we can replace all completions along the punctured line $\mathbf{C}^{\times} \cdot v$ by completions along the entire leaf $X_{\Gamma_{0}}$ (more generally, we can complete not merely along a punctured line but along any $\mathbf{C}^{\times}$-invariant locally closed subset which does not intersect its images under $N\left(\Gamma_{0}\right)^{0}$.)

\subsection{Quantization}

A standard quantization of $V$ is the algebra of differential operators on a Lagrangian $U \subseteq V$ (so that $V \cong T^{*} U$ ). So $X$ is quantized by $\mathcal{D}_{\hbar}(U)^{\Gamma}$. Call this $A_{X}$, and similarly define $A_{X_{\Gamma_{0}}}$ and $A_{V^{\Gamma_{0}} / \Gamma_{0}}$. By a straightforward quantum generalization of Proposition 53, one obtains, under the same assumptions:

Corollary 58. There is a canonical graded continuous $\mathbf{C} \llbracket \hbar \rrbracket$-algebra isomorphism

$$
\left(\hat{A}_{X}\right)_{\mathbf{C}^{\times} \cdot \bar{v}} \cong\left(\hat{A}_{X_{\Gamma_{0}}}\right)_{\mathbf{C}^{\times} \cdot \bar{v}} \hat{\otimes}_{\mathbf{C} \llbracket \hbar \rrbracket} A_{V^{\Gamma_{0}} / \Gamma_{0}} .
$$

This implies a positive answer to Question 51 for these quantizations.

There is a well-known family of quantizations which generalizes the invariant differential operators (and in fact yields the universal deformation of these), called spherical symplectic reflection algebras [EG02]. In order to be consistent with typical notation for symplectic reflection algebras, we will actually consider the Poisson variety $V^{*} / \Gamma$, so that its algebra of functions is $\operatorname{Sym}(V)^{\Gamma}$.

Recall that one constructs the universal deformation of $\operatorname{Weyl}(V) \rtimes \Gamma$ as follows ([EG02], cf. e.g., [Los12]). Let $S \subseteq \Gamma$ be the subset of symplectic reflections, i.e., $s \in S$ if $\operatorname{rk}(s-\mathrm{Id})=2$ (this is the minimal possible nonzero rank since the determinant of $s$ is one; geometrically, $s$ can be thought of as a generalized reflection around the symplectic subspace $\operatorname{ker}(s-\mathrm{Id}))$. Let $S=S_{1} \sqcup S_{2} \sqcup \cdots \sqcup S_{r}$ be the partition into conjugacy classes. Define the vector space $\mathfrak{c}$ with basis $\hbar, c_{1}, \ldots, c_{r}$, and for $s \in S_{i}$, we set $c(s):=c_{i}$. (Note that $\mathfrak{c}=\mathbf{C} \cdot \hbar \oplus \operatorname{Hom}_{\Gamma}(S, \mathbf{C})^{*}$.) Then we define the algebra $H(V, \Gamma)$ as the quotient of the skew-product algebra $\operatorname{Sym}(\mathfrak{c}) \otimes T V \rtimes \Gamma$ by the relations

$$
[x, y]=\hbar \omega(x, y)+\sum_{s \in S} c(s) \omega_{s}(x, y) s,
$$

where $\omega$ is the symplectic form, and $\omega_{s}$ is the projection of $\omega$ to $\wedge^{2} k e r(s-\mathrm{Id})^{\perp}$, i.e., $\omega_{s}(x, y)=$ 0 if $x$ or $y$ is in $\operatorname{ker}(s-\mathrm{Id})$, and $\omega_{s}(x, y)=\omega(x, y)$ if $x, y \in \operatorname{im}(s-\mathrm{Id})$.

Then, the (universal) spherical symplectic reflection algebra is defined as $U(V, \Gamma):=$ $e H(V, \Gamma) e$, where $e:=\frac{1}{|\Gamma|} \sum_{\gamma \in \Gamma} \gamma \in \mathbf{C}[\Gamma]$ is the symmetrizer. The algebra $U(V, \Gamma)$ encodes the family of quantizations $\hat{U}_{\lambda}(V, \Gamma)$ for $\lambda=\left(\lambda_{1}, \ldots, \lambda_{r}\right) \in \mathbf{C}^{r}$, where $U_{\lambda}(V, \Gamma):=$ 
$U(V, \Gamma) /\left(c_{i}-\lambda_{i} \hbar\right)$ and $\hat{U}_{\lambda}(V, \Gamma)$ is its $\hbar$-adic completion. There are canonical quotients $H(V, \Gamma) \rightarrow \mathcal{O}\left(V^{*}\right) \rtimes \Gamma$ and $U(V, \Gamma) \rightarrow \mathcal{O}\left(V^{*}\right)^{\Gamma}$, with kernel the ideal generated by $\mathfrak{c}$.

Then, Proposition 53 quantizes to the following statement. We will use the projection $\pi: H(V, \Gamma) \rightarrow \mathcal{O}\left(V^{*}\right) \rtimes \Gamma$ and similarly $\pi: U(V, \Gamma) \rightarrow \mathcal{O}\left(V^{*}\right)^{\Gamma}$. Given any closed subvariety $Z \subseteq V^{*} / \Gamma$, with ideal $I_{Z}$, let $\hat{H}(V, \Gamma)_{Z}$ denote the completion of $H(V, \Gamma)$ along the (two-sided) ideal generated by $\pi^{-1}\left(I_{Z}\right)$, i.e., $\pi^{-1}\left(\mathcal{O}\left(V^{*}\right) I_{Z} \rtimes \Gamma\right)$. Define in the same way $\hat{U}(V, \Gamma)_{Z}$, which equals $e \hat{H}(V, \Gamma)_{Z} e$. Given an open affine subvariety $Y \subseteq Z$, write $Z$ as the complement of the vanishing of a function $f \in \mathcal{O}\left(V^{*}\right)^{\Gamma}$. We can define the localizations $H(V, \Gamma)\left[\pi^{-1}(f)^{-1}\right]$ and $U(V, \Gamma)\left[\pi^{-1}(f)^{-1}\right]$, obtained by inverting all elements in $\pi^{-1}(f)$ (these are Ore localizations with respect to the set $\left.\bigcup_{m \geq 1} \pi^{-1}\left(f^{m}\right)\right)$. Then we define $\hat{H}(V, \Gamma)_{Y}$ and $\hat{U}(V, \Gamma)$ as the completions of these localizations with respect to the ideals generated by $\pi^{-1}\left(I_{Z}\right)$.

Let $x \in V^{*}$ be a point whose stabilizer is $\Gamma_{0}<\Gamma$. Let $\bar{x} \in V^{*} / \Gamma$ be its image.

In the above context (which is significantly more delicate than the context merely of invariant differential operators, due to the deformed relations), Losev proved a decomposition theorem [Los12, Theorem 1.2.1]. Using the result of the previous section, we can obtain a graded version of his theorem. Assume as before that $\mathbf{C}^{\times}$acts freely on $\bar{x}$. Suppose that (up to reordering) $S_{1}, \ldots, S_{i}$ are the conjugacy classes of symplectic reflections which intersect $\Gamma_{0}$, and let $S_{1}^{0}, \ldots, S_{i}^{0}$ be their intersections with $\Gamma_{0}$. We then consider $U\left(\left(V^{\Gamma_{0}}\right)^{\perp}, \Gamma_{0}\right)$ to be a $\mathbf{C} \llbracket \hbar, c_{1}, \ldots, c_{i} \rrbracket$-algebra, defined using the conjugacy classes $S_{1}^{0}, \ldots, S_{i}^{0}$.

Theorem 61. There are graded continuous $\mathbf{C} \llbracket \hbar, c_{1}, \ldots, c_{r} \rrbracket$-algebra isomorphisms

$$
\begin{gathered}
\hat{U}(V, \Gamma)_{\mathbf{C}^{\times} \cdot \bar{x}} \cong \hat{U}\left(V^{\Gamma_{0}},\{1\}\right)_{\mathbf{C}^{\times} \cdot \bar{x}} \hat{\otimes}_{\mathbf{C} \llbracket \hbar \rrbracket} \hat{U}\left(\left(V^{\Gamma_{0}}\right)^{\perp}, \Gamma_{0}\right)_{0} \llbracket c_{i+1}, \ldots, c_{r} \rrbracket, \\
\hat{H}(V, \Gamma)_{\mathbf{C}^{\times} \cdot \bar{x}} \cong \hat{H}\left(V^{\Gamma_{0}},\{1\}\right)_{\mathbf{C}^{\times} \cdot \bar{x}} \hat{\otimes}_{\mathbf{C} \llbracket \hbar \rrbracket} \operatorname{Mat}_{\left|\Gamma / \Gamma_{0}\right|}\left(\hat{H}\left(\left(V^{\Gamma_{0}}\right)^{\perp}, \Gamma_{0}\right)_{0}\right) \llbracket c_{i+1}, \ldots, c_{r} \rrbracket .
\end{gathered}
$$

Here $\operatorname{Mat}_{r}(A)$ is the algebra of $r$ by $r$ matrices with coefficients in $A$ (i.e., $\operatorname{Mat}_{r}(\mathbf{C}) \otimes A$ ).

The theorem implies that Question 51 has a positive answer for every completed spherical symplectic reflection algebra $\hat{U}_{\lambda}(V, \Gamma)$.

Remark 64. The way that the matrix algebra above actually arises is by the centralizer construction: for any algebra $A$ with an action by $\Gamma_{0}<\Gamma$, one takes $\operatorname{End}_{A}\left(\operatorname{Fun}_{\Gamma_{0}}(\Gamma, A)\right)$, where $\operatorname{Fun}_{\Gamma_{0}}(\Gamma, A)$ is the right $A$-module of $\Gamma_{0}$-equivariant functions from $\Gamma$ to $A$. It is clear that $\operatorname{End}_{A}\left(\operatorname{Fun}_{\Gamma_{0}}(\Gamma, A)\right) \cong \operatorname{Mat}_{\left|\Gamma / \Gamma_{0}\right|}(A)$, but the isomorphism depends on a set of representatives of the right cosets $\Gamma_{0} \backslash \Gamma$. We apply this to $A=\hat{H}\left(\left(V^{\Gamma_{0}}\right)^{\perp}, \Gamma_{0}\right)_{0}$.

The proof of the above theorem is by checking that the arguments of [Los12] go through when one completes along the punctured line $\mathbf{C}^{\times} \cdot \bar{x}$ instead of at $\bar{x}$. In fact, the above statement is a corollary of a sheafified statement [Los12, Theorem 2.5.3], which gives a $\mathbf{C}^{\times}$equivariant isomorphism of sheaves of $\widehat{\operatorname{Sym} \mathfrak{c}} \times \Gamma$-algebras on the symplectic leaf containing $\bar{x}$. We note that in the case where $\Gamma<\mathrm{GL}(U)$ for $U \subseteq V$ a Lagrangian subspace (so $V \cong T^{*} W$ and hence $\mathrm{GL}(U)<\mathrm{Sp}(V)$, one can instead use the argument of [BE09], which is simpler, and again complete along the punctured line instead of the point. We omit further details. 


\section{The hypertoric case}

Let $T^{m}=\left(\mathbf{C}^{\times}\right)^{m}$ act linearly and faithfully on $\mathbf{A}^{n}$, and let $\mathfrak{t}^{m}:=$ Lie $T^{m}$. Associated to this is a Hamiltonian action of $T^{m}$ on the cotangent bundle $T^{*} \mathbf{A}^{n}$, with the moment map $\mu: T^{*} \mathbf{A}^{n} \rightarrow\left(\mathfrak{t}^{m}\right)^{*} \cong \mathbf{C}^{m}$. We consider the variety $X=\mu^{-1}(0) / / T^{m}$, called the affine hypertoric variety. This is also denoted by $T^{*} \mathbf{A}^{n} / / / / T^{m}$, the Hamiltonian reduction. We assume here that $\mu^{-1}(0) / / T^{m}$ admits a smooth symplectic resolution given by a Geometric Invariant Theory quotient, $\mu^{-1}(0) / / \chi^{T^{m}}=\operatorname{Proj} \bigoplus_{m \geq 0} \mathbf{C}\left[\mu^{-1}(0)\right]^{m \chi}$, for a suitable character $\chi: T^{m} \rightarrow \mathbf{C}^{\times}$; in other words, as we recall in the proof, the action of $T^{m}$ is given by a unimodular hyperplane arrangement.

Let us use the $\mathbf{C}^{\times}$-action given by dilations in the vector space $\mathbf{C}^{2 n}=T^{*} \mathbf{A}^{n}$; thus $k=2$ in the previous notation.

In this case, a much stronger statement than Theorem 34 holds: we have in fact an equivariant Darboux-Weinstein decomposition Zariski-locally, as we prove below.

Theorem 65. Let $X$ be a hypertoric cone as above, $Z$ be a symplectic leaf of $X$ other than the vertex, and $z \in Z$ a point. Then there is an open $\mathbf{C}^{\times}$-stable neighborhood $X^{\circ}$ of $z$ which splits as a product of $\mathbf{C}^{\times}$-Poisson varieties,

$$
X^{\circ} \cong Z^{\circ} \times S
$$

with $Z^{\circ}=X^{\circ} \cap Z$ and $S$ the hypertoric cone corresponding to the slice of $Z$.

Moreover, $Z^{\circ}$ is $\mathbf{C}^{\times}$-Poisson isomorphic to the complement in $\mathbf{A}^{\operatorname{dim} Z}$ of $\leq n$ linear hyperplanes, equipped with the standard symplectic form, each of the coordinate functions homogeneous (not necessarily of degree one), and the symplectic form having weight two.

As we will see, the hyperplanes appearing in the second paragraph consist of some of the coordinate hyperplanes and at most $m / 2$ additional linear hyperplanes. We note that we use the notation $Z$ for the symplectic leaf in the theorem (and proof below) so as to be able to use coordinates $x_{i}, y_{i}$ on $T^{*} \mathbf{A}^{n}$ without confusion.

The theorem implies that Question 3 has an affirmative answer in this case.

Remark 66. For the above theorem, we did not need to assume that $\mathbf{C}^{\times}$acts freely on $z$, nor even that the stabilizer of $z$ be minimal in $Z$. This is a special feature of the hypertoric case (already in the finite linear quotient case, it follows from the preceding section that there need not be a product decomposition when $\mathbf{C}^{\times}$acts nonfreely, but rather one only gets a statement as in Theorem 34,(iv)). Note that, for generic $z \in Z$, the order of the stabilizer in $\mathbf{C}^{\times}$is either 1 or 2 , just as in the case of finite linear quotients (so the $\ell$ appearing in Theorem 34 will be either 1 or 2).

Remark 67. Recall that there is an important residual Hamiltonian action of $\bar{T}_{X}:=$ $\left(\mathbf{C}^{\times}\right)^{n} / T^{m}$ on $X$, obtained from the standard action of $\left(\mathbf{C}^{\times}\right)^{n}$ on $\mathbf{A}^{n}$ and hence its Hamiltonian action on $T^{*} \mathbf{A}^{n}$. We can similarly define residual tori $\bar{T}_{Z}$ and $\bar{T}_{S}$ acting on $Z$ and $S$, respectively. It follows from the proof below that $X^{\circ}$ and $Z^{\circ}$ are stable under their residual actions. There is a canonical exact sequence $1 \rightarrow \bar{T}_{S} \rightarrow \bar{T}_{X} \rightarrow \bar{T}_{Z} \rightarrow 1$ (whose maps descend 
from the inclusion and projection of coordinates on $\mathbf{A}^{n}$ ). Then, the isomorphism of the theorem is compatible with the actions of $\bar{T}_{S}$, and the projection $X^{\circ} \rightarrow Z^{\circ}$ carries the action of $\bar{T}_{X}$ to that of $\bar{T}_{Z}$.

Remark 68. It follows from the proof below that one similarly has a decomposition for the total space $\mathcal{X}:=T^{*} \mathbf{A}^{n} / / T^{m}$ of the natural family $\bar{\mu}: \mathcal{X} \rightarrow\left(\mathfrak{t}^{m}\right)^{*}$ of (Poisson) deformations of $X$, with $\bar{\mu}$ the map which factors the moment map $\mu: T^{*} \mathbf{A}^{n} \rightarrow \mathcal{X} \stackrel{\bar{\mu}}{\rightarrow} \mathfrak{t}^{m}$ (so $X=\bar{\mu}^{-1}(0)$, and $X_{t}:=\bar{\mu}^{-1}(t)$ gives the natural deformation for $\left.t \in \mathfrak{t}^{m}\right) 6$ Namely, we define $\mathcal{X}^{\circ}$ in the same way as we define $X^{\circ}$ in the proof (except without intersecting with $\bar{\mu}^{-1}(0)$ ), and we obtain the $\mathbf{C}^{\times}$-Poisson decomposition

$$
\mathcal{X}^{\circ} \cong Z^{\circ} \times \mathfrak{t}^{*} \times \mathcal{S}
$$

with $\mathfrak{t}=$ Lie $T$ as defined in the proof below, $Z^{\circ}$ the same as in theorem, and $\mathcal{S}$ the natural deformation of the hypertoric variety $S$ appearing there. The Poisson structure on $\mathfrak{t}^{*}$ is zero, and its $\mathbf{C}^{\times}$action is the square of the dilation action on $\mathbf{C}^{\operatorname{dim} \mathfrak{t}} \cong \mathfrak{t}^{*}$ (i.e., giving the coordinate functions degree two). There is also a natural formula for the map $\bar{\mu}$ on the RHS compatible with the isomorphism, and the residual Hamiltonian torus actions are compatible as in the previous remark.

Proof of Theorem 65. By [BD00, 3.2,3.3], the assumption on the resolution of $X$ above is equivalent to the following unimodularity condition. Without loss of generality, assume that $T^{m}$ acts on standard coordinate functions $x_{1}, \ldots, x_{n}, y_{1}, \ldots, y_{n}$ of $T^{*} \mathbf{A}^{n}$ diagonally, i.e., by

$$
\left(c_{1}, \ldots, c_{m}\right) \cdot x_{i}=\prod_{j=1}^{m} c_{j}^{b_{i j}} x_{i}, \quad\left(c_{1}, \ldots, c_{m}\right) \cdot y_{i}=\prod_{j=1}^{m} c_{j}^{-b_{i j}} y_{i} .
$$

In other words, the columns of the $n \times m$-matrix $\left(b_{j i}\right)$ span the kernel of the matrix dual to the associated central hyperplane arrangement of $n$ hyperplanes in $\left(\mathbf{C}^{n-m}\right)^{*}$. The unimodularity condition is equivalent to the condition that all nonzero $m \times m$-minors are \pm 1 .

In coordinates $\mathfrak{t}^{m}=\mathbf{C}^{m}$ the moment map $\mu$ takes the form

$$
\mu\left(x_{1}, \ldots, y_{n}\right)_{i}=\sum_{j=1}^{n} b_{j i} x_{j} y_{j}
$$

Let $\mathrm{pr}: \mu^{-1}(0) \rightarrow X$ be the projection. The symplectic leaves of $X$ are given by certain subgroups $\hat{T}<T^{m}$, called parabolic subgroups, which are defined as those such that $\hat{T}=$ $\operatorname{Stab}(v)$ for some $v \in \mathbf{C}^{n}$. Given such a subgroup, let $F \subseteq\{1, \ldots, n\}$ be the subset of coordinates on which $\hat{T}$ acts nontrivially, so that $F^{c}$, the complement, is the subset on which $\hat{T}$ acts trivially. Henceforth, for every $H \subseteq\{1, \ldots, n\}$, we let $\mathbf{A}^{H}$ denote the coordinate subset corresponding to $H$; then $\mathbf{A}^{F^{c}} \subseteq \overline{\mathbf{A}}^{n}$ is the fixed locus of $\hat{T}$. Let $T:=T^{m} / \hat{T}$,

\footnotetext{
${ }^{6}$ We remark that the assumption that $X$ admits a symplectic resolution is equivalent to the statement that $X_{t}$ be smooth for generic $t$, and hence smooth affine symplectic.
} 
which acts on $\mathbf{A}^{F^{c}}$. One obtains the closure $\bar{Z}$ of a symplectic leaf $Z$ by the Hamiltonian reduction of $T^{*} \mathbf{A}^{F^{c}}$ by $T$, i.e., $\bar{Z}=T^{*} \mathbf{A}^{F^{c}} / / / / \hat{T}=\left(\mu^{-1}(0) \cap T^{*} \mathbf{A}^{F^{c}}\right) / / T$. This identifies with $\operatorname{pr}\left(T^{*} \mathbf{A}^{F^{c}} \cap \mu^{-1}(0)\right) \subseteq X$. As before, we will also let $\mathfrak{t}=\operatorname{Lie}(T)$ and $\hat{\mathfrak{t}}=\operatorname{Lie}(\hat{T})$.

It is a general fact that the closures of the symplectic leaves are of this form. There are only finitely many, as there are only finitely many parabolic subgroups of $T^{m}$. This therefore determines the symplectic leaves themselves: the leaf $Z$ is the complement in $\bar{Z}$ of all proper subsets which are the closures of symplectic leaves. However, multiple $\hat{T}$ can produce the same symplectic leaf. We will therefore assume that $\hat{T}$ is maximal for its leaf. Then, the leaf $Z$ is the image under pr of the union of the closed free $T$-orbits. (Moreover, under this assumption, one can easily check that this produces a bijection between maximal $\hat{T}$ and symplectic leaves; see [PW07, $§ 2]$, where this is explained in terms of $F \subseteq\{1, \ldots, n\}$, and the $F$ that occur in this way are the coloop-free flats, which are natural combinatorially defined subsets of $\{1, \ldots, n\}$. We will not use these facts here.)

Let $Z$ be a symplectic leaf, $\hat{T}<T^{m}$ be a (in fact, the unique) maximal corresponding parabolic subgroup, and $F \subseteq\{1, \ldots, n\}$ be the subset as above of coordinates on which $\hat{T}$ acts nontrivially. The closure $\bar{Z}$ is then given by $\bar{Z}=\left(\mu^{-1}(0) \cap T^{*} \mathbf{A}^{F^{c}}\right) / / T$. Let $z \in Z$ and let $\tilde{z} \in T^{*} \mathbf{A}^{F^{c}}$ be a preimage of $z$ in $\mu^{-1}(0) \cap T^{*} \mathbf{A}^{F^{c}}$.

Let $G \subseteq F^{c}$ be a subset with $|G|=\operatorname{dim} T$ such that $T$ acts with finite kernel on $\mathbf{A}^{G}$, such that for all $i \in G$, either $\tilde{z}\left(x_{i}\right) \neq 0$ or $\tilde{z}\left(y_{i}\right) \neq 0$. Such a subset must exist because $T \cdot \tilde{z}$ is a free orbit. Moreover, by our unimodularity hypothesis, the kernel of $T$ on $\mathbf{A}^{G}$, à priori finite, must actually be trivial; thus, $T$ acts generically faithfully on $\mathbf{A}^{G}$. Without loss of generality (up to swapping some of the $x_{i}$ with $y_{i}$ ), we can actually assume that $\tilde{z}\left(x_{i}\right) \neq 0$ for all $i \in G$.

We then define $\left(T^{*} \mathbf{A}^{n}\right)^{\circ}$ as the complement of coordinate hyperplanes on which $\tilde{z}$ is nonzero. We similarly define $\left(T^{*} \mathbf{A}^{H}\right)^{\circ}:=T^{*} \mathbf{A}^{H} \cap\left(T^{*} \mathbf{A}^{n}\right)^{\circ}$ and $\left(\mathbf{A}^{H}\right)^{\circ}:=\mathbf{A}^{H} \cap\left(T^{*} \mathbf{A}^{n}\right)^{\circ}$ for all $H \subseteq\{1, \ldots, n\}$. In particular, $\left(\mathbf{A}^{G}\right)^{\circ}$ is the locus where all coordinates are nonzero, and $T$ acts freely and transitively on it. It follows that the $T$-orbits in $\left(T^{*} \mathbf{A}^{F^{c}}\right)^{\circ}$, or equivalently the $T^{m}$-orbits, are all closed and free, and that $Z^{\circ}=\operatorname{pr}\left(\left(T^{*} \mathbf{A}^{F^{c}}\right)^{\circ} \cap \mu^{-1}(0)\right)$. (We remark that, if one desired, one could alternatively have defined $\left(T^{*} \mathbf{A}^{F^{c}}\right)^{\circ}$ to be the maximal subset consisting of closed $T$-orbits such that the $x_{i}$ coordinate is nonzero for all $i \in G$; this is a larger open subset and depends only on $G$ and $Z$ and not on $z$, but has the disadvantage of not being affine. We could similarly consider any (affine) open $T$-stable subset thereof. We would then set $T^{*} \mathbf{A}^{n}=\left(T^{*} \mathbf{A}^{F^{c}}\right)^{\circ} \times T^{*} \mathbf{A}^{F}$.)

Next, let us restrict the moment map to $T^{*}\left(\mathbf{A}^{F^{c}}\right)^{\circ}$. This lands in $\mathfrak{t}^{*} \subseteq\left(\mathfrak{t}^{m}\right)^{*}$. By the above, for every $z \in \mathfrak{t}^{*}$, the equations cutting out $\mu^{-1}(z)$ in $\left(\mathbf{A}^{F^{c}}\right)^{\circ}$ uniquely solve for $x_{i} y_{i}, i \in G$ in terms of $x_{j} y_{j}, j \in F^{c} \backslash G$. In other words, since $x_{i}$ are invertible on $\left(\mathbf{A}^{F^{c}}\right)^{\circ}$ for $i \in G$, these equations uniquely solve for $y_{i}, i \in G$ in terms of the other coordinates. Hence, we have an isomorphism

$$
\pi^{F^{c} \backslash G} \times \mu \times \pi^{\prime}: T^{*}\left(\mathbf{A}^{F^{c}}\right)^{\circ} \stackrel{\sim}{\rightarrow}\left(T^{*} \mathbf{A}^{F^{c} \backslash G}\right)^{\circ} \times \mathfrak{t}^{*} \times\left(\mathbf{A}^{G}\right)^{\circ},
$$

with $\pi^{F^{c} \backslash G}$ the projection to $T^{*} \mathbf{A}^{F^{c} \backslash G}$ and $\pi^{\prime}$ the projection to $\mathbf{A}^{G} \subseteq T^{*} \mathbf{A}^{G}$ (just the $x$ coordinates). Since $\left(\mathbf{A}^{G}\right)^{\circ} \cong T$ consists of a single free $T$-orbit and $\left(T^{*} \mathbf{A}^{F^{c}}\right)^{\circ}$ is affine, the above produces an isomorphism $\left(T^{*} \mathbf{A}^{F^{c} \backslash G}\right)^{\circ} \cong Z^{\circ}$. 
The final assertion then follows immediately. The weights of the coordinate functions $x_{i}, y_{i}, i \in F^{c} \backslash G$ on $T^{*} \mathbf{A}^{F^{c} \backslash G} \cong \mathbf{A}^{\operatorname{dim} Z}$ are then explicitly given as follows: for each $i \in$ $F^{c} \backslash G$, let $r_{i j} \in \mathbf{Z}$ be the unique integers such that $x_{i} \prod_{j \in G} x_{j}^{r_{i j}}$ is $T$-invariant. Then, $\left|x_{i}\right|:=\left|x_{i} \prod_{j \in G} x_{j}^{r_{i j}}\right|=1+\sum_{j \in G} r_{i j}$, and similarly we compute the degrees of the $y_{i}$.

Next, write $T^{m}=T^{\prime} \times \hat{T}$ for $T^{\prime} \subseteq T^{m}$ some connected subtorus such that the composition $T^{\prime} \rightarrow T^{m} \rightarrow T$ is an isomorphism. Let $\mu_{T^{\prime}}$ and $\mu_{\hat{T}}$ be the restricted moment maps, valued in $\left(\mathfrak{t}^{\prime}\right)^{*}$ and $\hat{\mathfrak{t}}^{*}$, respectively. Then $\mu^{-1}(0)=\mu_{\mathfrak{t}^{\prime}}^{-1}(0) \cap \mu_{\hat{\mathfrak{t}}}^{-1}(0)$. Let $\pi^{F}: T^{*} \mathbf{A}^{n} \rightarrow T^{*} \mathbf{A}^{F}$ be the projection. Then the projection to $T^{*} \mathbf{A}^{F^{c}}$ produces an isomorphism

$$
\left(\pi^{F}\right)^{-1}(z) \cap \mu_{\mathfrak{t}^{\prime}}^{-1}(0) \cong \mu^{-1}\left(-\mu_{\mathfrak{t}^{\prime}}(z)\right) \cap T^{*} \mathbf{A}^{F^{c}},
$$

where we use in the first term on the RHS the isomorphism $\mathfrak{t}^{\prime} \cong \mathfrak{t}$. Thus, restricted to $\left(T^{*} \mathbf{A}^{n}\right)^{\circ} \cap \mu_{\mathfrak{t}^{\prime}}^{-1}(0), \pi^{F}$ is a fibration with fibers isomorphic to $T^{*} \mathbf{A}^{F^{c} \backslash G} \times\left(\mathbf{A}^{G}\right)^{\circ}$. We conclude that $\pi^{F} \times \pi^{F^{c} \backslash G} \times \pi^{\prime}$ induces an isomorphism

$$
\mu^{-1}(0) \cap\left(T^{*} \mathbf{A}^{n}\right)^{\circ} \cong\left(\pi^{F} \times \pi^{F^{c} \backslash G} \times \pi^{\prime}\right)\left(\mu_{\hat{\mathfrak{t}}}^{-1}(0)\right) \cap\left(T^{*} \mathbf{A}^{F} \times\left(T^{*} \mathbf{A}^{F^{c} \backslash G}\right)^{\circ} \times\left(\mathbf{A}^{G}\right)^{\circ}\right) .
$$

Now, $\mu_{\hat{\mathfrak{t}}}$ is trivial in the $T^{*} \mathbf{A}^{F^{c}}$ direction. That is, for $\mu_{\hat{\mathfrak{t}}}^{F}: T^{*} \mathbf{A}^{F} \rightarrow \hat{\mathfrak{t}}$ the restricted moment map, we have $\mu_{\hat{\mathfrak{t}}}=\mu_{\hat{\mathfrak{t}}}^{F} \circ \pi^{F}$. We conclude that

$$
\left(T^{*} \mathbf{A}^{n}\right)^{\circ} \cap \mu^{-1}(0) \cong\left(\left(\mu_{\hat{\mathfrak{t}}}^{F}\right)^{-1}(0) \cap T^{*} \mathbf{A}^{F}\right) \times\left(T^{*} \mathbf{A}^{F^{c} \backslash G}\right)^{\circ} \times\left(\mathbf{A}^{G}\right)^{\circ}
$$

Moreover, given functions on any two of the above three factors, if we write them in terms of standard coordinate functions, the resulting functions on $T^{*} \mathbf{A}$ Poisson-commute. By the unimodularity condition, the action of $T$ on $\left(\mathbf{A}^{G}\right)^{\circ}$ is free and transitive. Therefore, we can replace each coordinate function $x_{i}$ or $y_{i}$ on $T^{*} \mathbf{A}^{F}$ with its product by a monomial $x_{i}^{\prime}:=x_{i} \prod_{j \in G} x_{j}^{r_{j}}$ (or similarly $y_{i}^{\prime}:=y_{i} \prod_{j \in G} x_{j}^{s_{j}}$ ) so that $x_{i}^{\prime}, y_{i}^{\prime}$ are invariant under $T^{\prime}$, where here the $x_{j}$ are the coordinate functions in the $\mathbf{A}^{n}$ direction (hence when $j \in G$, they are coordinates on $\mathbf{A}^{G}$ ). The new $x_{i}^{\prime}, y_{i}^{\prime}$ still commute with all functions on $T^{*} \mathbf{A}^{F^{c} \backslash G} \times \mathbf{A}^{G}$ when written in terms of standard coordinate functions. Therefore, restricting to $\mu^{-1}(0)$ and quotienting by $T^{m}$ yields the $\mathbf{C}^{\times}$-equivariant Poisson isomorphism

$$
\left(T^{*} \mathbf{A}\right)^{\circ} / / / / T^{m} \cong Z^{\circ} \times S
$$

\subsection{Quantization}

Since we have a Zariski-local decomposition, we can immediately conclude a quantization of $X^{\circ}$. Let $A_{Z}$ and $A_{X}$ be the standard deformation quantizations of the hypertoric cones $Z$ and $S$. Namely, these are given by quantum Hamiltonian reduction $A_{Z}=\mathcal{D}_{\hbar}\left(A^{F^{c}}\right) / / / T:=$ $\left(\mathcal{D}_{\hbar}\left(\mathbf{A}^{F^{c}}\right) / \mu_{q, F^{c}}(\mathfrak{t}) \mathcal{D}\left(\mathbf{A}^{F^{c}}\right)\right)^{T}$, with $\mu_{q, F^{c}}: \mathfrak{t} \rightarrow \mathcal{D}_{\hbar}\left(\mathbf{A}^{F^{c}}\right)$ the quantum comoment map with $\mu_{q, F^{c}}(\xi)$ the vector field given by the action of $\xi$, viewed as a differential operator. Similarly define $A_{S}$ and $A_{X}$. Since $X^{\circ}$ is the complement in $X$ of the images of some linear hyperplanes, we can define the corresponding localization $A_{X^{\circ}}$ by inverting the corresponding linear functions and taking the $\hbar$-adic completion. Note that $Z^{\circ}=Z \cap X^{\circ}$; thus $A_{Z^{\circ}}$ 
is obtained from $A_{Z}$ by inverting the same coordinate functions and completing. We equip these algebras with the Euler vector field which assigns the coordinate functions the same degrees as in the graded algebras $\mathcal{O}(S), \mathcal{O}(Z)$, and $\mathcal{O}(X)$. Then $A_{Z^{\circ}} \hat{\otimes}_{\mathbf{C} \llbracket \hbar \rrbracket} A_{S}$ is a deformation quantization of $X^{\circ}$, which is $\mathbf{C}^{\times}$-compatible by Theorem 65. (Note that $\mathcal{O}(Z)$ and $\mathcal{O}(S)$ are not, in general, nonnegatively graded; neither is, obviously, $A_{X^{\circ}}$ nor $A_{Z^{\circ}}$. Thus, the quantizations are not completed Rees algebras of nonnegatively filtered algebras.)

Theorem 70. We have an isomorphism of $\mathbf{C}^{\times}$-compatible deformation quantizations,

$$
A_{X^{\circ}} \cong A_{Z^{\circ}} \hat{\otimes}_{\mathrm{C} \llbracket \hbar \rrbracket} A_{S} .
$$

The fact that both sides of the isomorphisms above are quantizations of $X^{\circ}$ is immediate; to prove the two are isomorphic follows from a straightforward quantum analogue of the proof of Theorem [65. We note that the theorem yields a positive answer to Question 51 in the case of the quantization $A_{X^{\circ}}$.

Remark 72. In the previous section, we considered the universal deformation of algebras of invariant differential operators, given by spherical symplectic reflection algebras. One can similarly form the universal family of (graded) deformation quantizations of the quantized hypertoric varieties $A_{X}$ above, by performing quantum Hamiltonian reduction not merely at the 0 character but at arbitrary characters of $\mathfrak{t}^{m}$ (these were considered, for example, in [BLPW12]). Then, the above theorem goes through for these as well: given a character $\zeta \in\left(\mathfrak{t}^{m}\right)^{*}$, one considers the restriction $\left.\zeta\right|_{\hat{\mathfrak{t}}}$ as in the proof of Theorem 65, and obtains the $\mathbf{C}^{\times}$-equivariant (continuous $\mathbf{C} \llbracket \hbar \rrbracket$-algebra) isomorphism:

$$
A_{X^{\circ}}^{\hbar \zeta} \cong A_{Z^{\circ}} \hat{\otimes}_{\mathbf{C} \llbracket \hbar \rrbracket} A_{S}^{\hbar \zeta \mid \hat{\mathfrak{t}}_{\hat{\mathfrak{t}}}}
$$

where $A_{X}^{\hbar \zeta}:=\left(\mathcal{D}_{\hbar}\left(\mathbf{A}^{n}\right) /\left(\mu_{q}(t)-\hbar \zeta(t)\right)_{t \in \mathfrak{t}^{m}} \mathcal{D}_{\hbar}\left(\mathbf{A}^{n}\right)\right)^{T^{m}}, A_{X^{\circ}}^{\hbar \zeta}$ is its completed localization, and we similarly define $A_{S}^{\zeta \mid \hat{t}}$. Note that we need not deform $A_{Z^{\circ}}$; this makes sense since $Z^{\circ}$ is just the complement of some hyperplanes in a symplectic affine space. We conclude that Question 51 has a positive answer in the case of these quantizations. (One can also put the deformations together into a family and produce an isomorphism resembling that of Remark 68 and analogous to Theorem 61).

Remark 74. It would also be interesting to prove a sheafified version of the decomposition, parallel to the decomposition of [Los12, Theorem 2.5.3], which produces a global statement for sheaves on $Z$ of completed quantizations, and to study how the decomposition varies as $z$ (or the line $\mathbf{C}^{\times} \cdot z$ ) varies.

\section{References}

[BD00] R. Bielawski and A. S. Dancer, The geometry and topology of toric hyperkähler manifolds, Comm. Anal. Geom. 8 (2000), no. 4, 727-760. MR 1792372 
[BDMN] G. Bellamy, C. Dodd, K. McGerty, and T. Nevins, Categorical cell decomposition of quantized symplectic algebraic varieties, arXiv:1311.6804.

[BE09] R. Bezrukavnikov and P. Etingof, Parabolic induction and restriction functors for rational Cherednik algebras, Selecta Math. (N.S.) 14 (2009), no. 3-4, 397-425. MR 2511190 (2010e:20007)

[Bea00] A. Beauville, Symplectic singularities, Invent. Math. 139 (2000), no. 3, 541-549, arXiv:math/9903070. MR MR1738060 (2001f:14008)

[Bel09] G. Bellamy, On singular Calogero-Moser spaces, Bull. Lond. Math. Soc. 41 (2009), no. 2, 315-326, arXiv:0707.3694. MR MR2496507 (2010h:14022)

[BLPW] T. Braden, A. Licata, N. Proudfoot, and B. Webster, Quantizations of conical symplectic resolutions II: category $O$, arXiv:1407.0964.

[BLPW12] _ Hypertoric category O Adv. Math. 231 (2012), no. 3-4, 1487-1545, arXiv:1010.2001. MR 2964613

[BPW12] T. Braden, N. Proudfoot, and B. Webster, Quantizations of conical symplectic resolutions I: local and global structure, arXiv:1208.3863, 2012.

[BS13] G. Bellamy and T. Schedler, On the (non)existence of symplectic resolutions for imprimitive symplectic reflection groups, arXiv:1309.3558, 2013.

[EG02] P. Etingof and V. Ginzburg, Symplectic reflection algebras, Calogero-Moser space, and deformed Harish-Chandra homomorphism, Invent. Math. 147 (2002), no. 2, 243-348. MR MR1881922 (2003b:16021)

[ES10] P. Etingof and T. Schedler, Poisson traces and $\mathcal{D}$-modules on Poisson varieties, Geom. Funct. Anal. 20 (2010), no. 4, 958-987, arXiv:0908.3868, with an appendix by I. Losev.

[ES12] Z Z Zeroth Poisson homology of symmetric powers of isolated quasihomogeneous surface singularities, J. Reine Angew. Math. 667 (2012), 67-88, arXiv:0907.1715. MR 2929672

[ES16] _ Coinvariants of Lie algebras of vector fields on algebraic varieties, accepted to Asian J. Math, arXiv:1211.1883, 2016.

[Fer70] A. Ferrari, Cohomology and holomorphic differential forms on complex analytic spaces, Ann. Scuola Norm. Sup. Pisa (3) 24 (1970), 65-77. MR 0274810

[Fer71] C Coomologia e forme differenziali sugli spazi analitici complessi, Ann. Scuola Norm. Sup. Pisa (3) 25 (1971), 469-480. MR 0414922

[Har72] R. Hartshorne, Algebraic de Rham cohomology, Manuscripta Math. 7 (1972), 125-140. MR 0313255 (47 \#1810) 
[Har75] On the De Rham cohomology of algebraic varieties, Inst. Hautes Études Sci. Publ. Math. (1975), no. 45, 5-99. MR 0432647 (55 \#5633)

[Kal06] D. Kaledin, Symplectic singularities from the Poisson point of view, J. Reine Angew. Math. 600 (2006), 135-156. MR MR2283801 (2007j:32030)

[Kal09] Geometry and topology of symplectic resolutions, Algebraic geometrySeattle 2005. Part 2, Proc. Sympos. Pure Math., vol. 80, Amer. Math. Soc., Providence, RI, 2009, arXiv:math/0608143, pp. 595-628. MR 2483948 (2010a:14023)

[Lam99] T. Y. Lam, Lectures on modules and rings, Graduate Texts in Mathematics, vol. 189, Springer-Verlag, New York, 1999. MR 1653294 (99i:16001)

[Los10] I. Losev, Quantized symplectic actions and $W$-algebras, J. Amer. Math. Soc. 23 (2010), no. 1, 35-59. MR MR2552248

[Los12] _ Completions of symplectic reflection algebras, Selecta Math. (N.S.) 18 (2012), no. 1, 179-251, arXiv:1001.0239, MR 2891864

[Nam13a] Y. Namikawa, Equivalence of symplectic singularities, Kyoto J. Math. 53 (2013), no. 2, 483-514. MR 3079311

[Nam13b] — Fundamental groups of symplectic singularities, arXiv:1301.1008, 2013.

[Nam14] _ A finiteness theorem on symplectic singularities, arXiv:1411.5585, 2014.

[PS16] N. Proudfoot and T. Schedler, Poisson-de Rham homology of hypertoric varieties and nilpotent cones, arXiv:1405.0743, to appear in Selecta Mathematica, DOI 10.1007/s00029-016-0232-3, 2016.

[PW07] N. Proudfoot and B. Webster, Intersection cohomology of hypertoric varieties, J. Algebraic Geom. 16 (2007), no. 1, 39-63. MR 2257319 (2008m:14037)

[Wei83] A. Weinstein, The local structure of Poisson manifolds, J. Differential Geom. 18 (1983), no. 3, 523-557.

[Xu14] C. Xu, Finiteness of algebraic fundamental groups, Compos. Math. 150 (2014), no. 3, 409-414. MR 3187625 